\title{
Radar imaging of spheres in 3D using MUSIC
}

D. H. Chambers and J. G. Berryman

January 21, 2003

U.S. Department of Energy

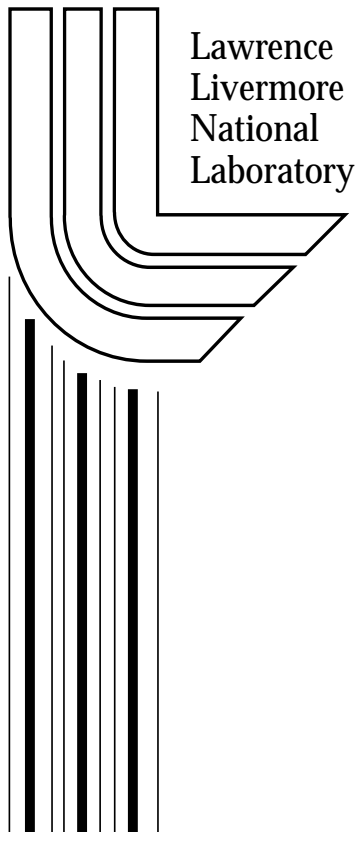




\section{DISCLAIMER}

This document was prepared as an account of work sponsored by an agency of the United States Government. Neither the United States Government nor the University of California nor any of their employees, makes any warranty, express or implied, or assumes any legal liability or responsibility for the accuracy, completeness, or usefulness of any information, apparatus, product, or process disclosed, or represents that its use would not infringe privately owned rights. Reference herein to any specific commercial product, process, or service by trade name, trademark, manufacturer, or otherwise, does not necessarily constitute or imply its endorsement, recommendation, or favoring by the United States Government or the University of California. The views and opinions of authors expressed herein do not necessarily state or reflect those of the United States Government or the University of California, and shall not be used for advertising or product endorsement purposes.

This work was performed under the auspices of the U. S. Department of Energy by the University of California, Lawrence Livermore National Laboratory under Contract N o. W-7405-Eng-48.

This report has been reproduced directly from the best available copy.

A vailable electronically at http:/ / www.doc.gov/ bridge

A vailable for a processing fee to U.S. Department of Energy

And its contractors in paper from

U.S. Department of Energy

Office of Scientific and Technical Information

P.O. Box 62

Oak Ridge, TN 37831-0062

Telephone: (865) 576-8401

Facsimile: (865) 576-5728

E-mail: reports@adonis.osti.gov

A vailable for the sale to the public from

U.S. Department of Commerce

National Technical Information Service

5285 Port Royal Road

Springfield, VA 22161

Telephone: (800) 553-6847

Facsimile: (703) 605-6900

E-mail: orders@ntis.fedworld.gov

Online ordering: http:/ / www.ntis.gov/ ordering.htm

OR

Lawrence Livermore N ational Laboratory

Technical Information Department's Digital Library

http:/ / www.Ilnl.gov/ tid/ Library.html 


\title{
Radar imaging of spheres in 3D using MUSIC
}

\author{
D. H. Chambers and J. G. Berryman ${ }^{1}$ \\ Lawrence Livermore National Laboratory \\ Livermore, CA 94551-9900
}

\section{INTRODUCTION}

The use of Synthetic Aperture Radar (SAR) imaging techniques to find targets partially covered by trees and foliage canopies has been extensively studied in recent years (Theron et al. 1999; Sarabandi and Koh 2001; Xu and Narayanan 2001a; Xu and Narayanan 2001b). Earlier studies included analysis of field data (Durden et al. 1991; Durden et al. 1993; Fleischman et al. 1996; Toups et al. 1996; Fleischman et al. 1996) and relevant EM code development (Senior et al. 1987; Ulaby et al. 1990). The present paper will describe a new approach to these problems, and describe some simulation results relevant to the development of these ideas into a tool for both fixed and synthetic aperture imaging of targets using electromagnetic waves.

Most of the discipline of geophysics is devoted to making physical measurements of the earth's interior and the earth's surface using seismic, electrical, electromagnetic, or other methods (e.g., various logging tools) to determine the internal structure and surface features of the earth, and perhaps more to the point - how the materials and structure are distributed spatially in specific regions of interest. Since variations of materials are ubiquitous, occurring at all places and all scales of interest, the general earth imaging problem is very difficult: it invariably requires more data and, therefore, more data processing, than we can normally afford in order to do the job "right." It would therefore be most helpful if there were some means for skipping over those parts of the earth environment that we do not really want to image very much, and looking at those parts (which we will term "targets") that do interest us. This sounds challenging (maybe even impossible), but recent developments in acoustics make it clear that certain very limited imaging goals are achievable with much smaller data sets than are traditionally needed in, for example, seismic array processing (Claerbout 1976). Early versions of this new method has been given the names of "time-reversal acoustics" or "time-reversal mirrors," and have been developed most extensively by the French ultrasonics group led by Fink [see Prada et al. (1991), Prada and Fink (1994), Prada et al. (1995), Prada et al. (1996), Fink (1997), Fink (1999), Fink and Prada (2001), etc.].

Although these new ideas about target localization and imaging first arose in acoustics applications, the basic ideas are all wave-based and, therefore, pertinent with only a few changes to electromagnetic imaging modalities as well. The basic idea behind time-reversal acoustics is this: If we have an acoustic medium that has a few isolated targets (say a harbor with boats, buoys, and

\footnotetext{
${ }^{1}$ Research supported by DARPA
} 
mines), then by using a fairly arbitrarily structured acoustic array, we can train the array to send sound preferentially to the main scattering targets. Thus, the array will become self-focusing. We do this by first sending out an initial pulse from any one, or some subset, of the array elements. The pulse propagates out in a spherical wavefront in all directions, and scattered energy returns from any obstacle encountered. When the return signal (back-scattered from the main targets) arrives at the array elements, we record the entire wavetrain (for some finite period of time that depends on our goals). Then, we time-reverse (last in, first out) the wavetrain and send the entire time-reversed wavetrain back into the acoustic medium. This procedure will produce a new return signal, one that we record and then re-emit in reversed time sequence. The iteration process has the effect of increasing the wavefield amplitude from scattering obstacles without either needing or providing detailed information about the background medium properties. We continue this process until it converges so that the returned signal is the same (within some tolerance) as the one received previously. What this process has accomplished empirically is to find the eigenfunction (eigenvector for a finite discrete array) of the scattering operator with the largest eigenvalue. This is precisely the well-known power method for finding the eigenvector associated with the largest eigenvalue of the operator.

Now this empirical method of doing time-reversal processing has certain advantages for applications in very complex media, because its success depends only on the general principle of reciprocity in wave propagation problems. Of greatest importance, it does not depend at all on the propagating medium being homogeneous. If, for biomedical applications, we wanted to do this type of processing and proceed to turn up the volume of the ultrasound for one final iteration, this can be used to blast and disintegrate the main target. Such methods have been used successfully for example to eliminate kidney stones [an approach known as lithotripsy (Thomas et al. 1996; Fink 1997)] and other methods of acoustic surgery (ter Haar 2001) could also be advanced by using time-reversal techniques for wave focusing.

However, for localization (or imaging) of targets, another approach using the same fundamental ideas as those behind the methods of physical time-reversal is cheaper (because it requires less specialized hardware) and more effective. Instead of time-reversing and iterating the signals in the scattering process, we can map out once and for all the full response (or transfer) matrix. First, we excite any one of the array elements as a source and record the received scattered signal (timetrace) at all array elements. (Each array element is considered to be both a source and a receiver for seismic waves.) Then repeat this process using each array element in turn as a source until we have filled in the entire $N \times N$ response matrix (for an $N$ element array). By Fourier transforming the elements of this temporal matrix, we obtain a frequency indexed set of complex but symmetric matrices. For each of these matrices we can perform the well-known singular value decomposition (SVD). Thus, we find all the desired information (all the singular vectors and singular values) simultaneously for each frequency. Then we can order the singular vectors, find the largest, next largest, etc. If there are $M$ targets and $M<N$, then it is known that there will be $M$ or more "significant" singular values associated with the targets, and the remainder will be zero or very close to zero. By using our knowledge of how sound propagates in a 3D homogeneous medium, we can then use this information to locate all $M$ of the targets (approximately, but this is the best that can be done with this method). One application is locating a few large targets within a large region that could be investigated in detail later, using a high resolution survey method, once the approximate number and location of targets has been determined. Another example from electromagnetics is an array of EM antennas around the perimeter of a compound to be defended 
against intruders.

The response matrix method clearly generalizes to electromagnetic imaging, because the only physical requirement for the success of this approach is the validity of reciprocity, which is true for all the linear equations for wave propagation for stationary media in mathematical physics. There are, however, important details especially pertinent to the EM applications that need to be addressed: (1) In acoustics the signals are pressure waves and do not carry polarization information, whereas in electromagnetics the signals are always transversely polarized, and can therefore carry information about either of two states of polarization. So there should be some orientation information present in electromagnetic data that is not available in acoustic data. How this new information might be applied to the imaging problem is not known at present (although the present authors are currently studying this problem). (2) Forests and foliage are heterogeneous, so the localization/imaging will degrade as the degree of this inhomogeneity increases due to greater foliage density. Similar issues have been studied by Berryman and colleagues (Berryman et al. 2002; Borcea et al. 2002) at Stanford for the 2D acoustics problem, and that new method can be retooled for the EM problem. (3) The third issue is that time-reversal imaging has been applied mostly to relatively small objects, often treated as point scatterers. Chambers and Gautesen (2001), working at LLNL, have recently addressed this issue for the acoustics case, but not for EM problems. They found that individual scatterers can have multiple eigenvalues and eigenvectors associated with them, when more than one of the physical properties of the objects show contrast with those of the surroundings (for example, bulk modulus and density for acoustics). However, the good news is that the additional eigenvalues are often much smaller than the one that corresponds to the physical quantity that gives rise to the eigenvalue for point scatterers (i.e., changes in bulk modulus). So we do not expect that multiple eigenvalues per target will cause any special problems for the EM case, but the issue is important and needs to be studied carefully which we do here. We should also stress that there can also be important advantages to having multiple eigenvalues and eigenvectors associated with a given target, because the additional information may be useful for target discrimination and identification.

Though imaging through foliage is our primary problem, the complicated multiple scattering, as well as ground effects (reflections and clutter), makes this a formidable problem to start with. Thus we chose to begin our investigations into the issues of EM imaging with the simpler case of dielectric spheres over a ground plane. We wish to use this simpler case to understand the differences between the acoustic case reported in previous studies, and EM imaging. In addition, we can begin to explore polarization issues and how to incorporate reflections from the ground. We wish to determine the number of eigenvalues of the response matrix required for optimal imaging and which variations of the MUSIC algorithm work best. This article reports on our work to date on imaging multiple spheres with a linear and a planar array using a single polarization. In section 2 we describe the sphere and array configuration that was used, and the method for obtaining simulated data. Section 3 describes the imaging methods and section 4 presents the results for 1, 2 , and 3 spheres.

\section{MULTIPLE SPHERE PROBLEM}

As mentioned previously, the application we wish to investigate is detection of objects in a forest by a radar array looking into the trees. Imaging through the trees is complicated by the multiple scattering from the tree trunks, as well as ground effects (reflections and clutter). In addition, there are the issues mentioned in the Introduction, such as polarization, in imaging with 
EM radiation that are not present in acoustic imaging. To investigate issues unique to EM imaging over the ground, we will consider the simpler problem of imaging multiple spheres with an array positioned above a ground plane. We employ an EM modeling code to generate simulated data that we process to form images. We choose a frequency of $1 \mathrm{GHz}$ (L-band), typical of imaging radars, and horizontal polarization. The latter choice simplifies the scattering from tree trunks for our application of imaging through a forest.

Our computational model consists of a source/receiver array of short dipoles arranged either in a single line parallel to the ground or in a plane normal to the ground, a number of scattering spheres, and a perfectly conducting ground plane to model reflections from damp earth in the subsoil. Each dipole element of the array is $7.5 \mathrm{~cm}$ long $(\lambda / 4)$ and oriented parallel to the ground plane, emitting horizontally polarized radiation. Two array configurations are considered, a 42 element linear array, and a 42 element planar array. The linear array consists of 21 transmit elements and 21 receive elements arranged in pairs, with each receive element placed $7.5 \mathrm{~cm}$ above its corresponding transmit element. The pairs are arranged in a line, $15 \mathrm{~cm}$ apart (center to center), for a total length of 3 meters $(10 \lambda)$. The line of transmit elements is placed at a height of 3 meters above the ground. In the planar array configuration, the transmit/receive pairs are arranged in three horizontal lines of 7 elements each. The separation between elements in each line is $30 \mathrm{~cm}$ $(1 \lambda)$ center-to-center, and the vertical separation between lines is $15 \mathrm{~cm}$. Thus the array aperture is 1.8 meters $(6 \lambda)$ horizontally and $30 \mathrm{~cm}$ vertically. The bottom line of transmitters is placed $2.85 \mathrm{~m}$ above the ground. The transmit elements are driven by specifying the voltage across an infinitesimal gap between the two halves of each dipole. The output of the simulator is the current through a $50 \Omega$ resistor placed across an infinitesimal gap between the two halves of each dipole receiver element.

The scattering spheres are $60 \mathrm{~cm}$ in diameter and given a relative dielectric constant typical of wood, $\varepsilon_{r}=27.3-8.4 i$ (Karam et al. 1992). These are placed at various positions in front of the array at ranges between 10 to 17 meters. The ground is modeled as a perfect conductor, reflecting all radiation.

For each array and sphere configuration a series of simulations are performed using EIGER, a spectral domain analysis code developed by Lawrence Livermore National Laboratory, Sandia National Laboratory, SPAWAR SSC San Diego, and the University of Houston (Champagne II et al. 2001; White et al. 2000). EIGER uses the method of moments to solve integral equations for the currents on surfaces of objects given voltages at various points on an object or an incident plane wave. Different integral equations can be specified depending on the boundary conditions on each surface (e.g., perfectly conducting, impedance boundary, dielectric material). Objects are specified as collections of surfaces and wires that are divided into discrete elements, with currents represented by basis functions defined on each element. For our simulations, dipole antennas were specified as thin wires (zero thickness), and spheres by surfaces between free space and dielectric. Each dipole was represented by six linear elements and each sphere by 100 surface elements with linear basis functions. For each transmit array element, the currents from the receivers were calculated given a unit transmit current. By repeating this for all transmit elements, a complex current response matrix for the array is obtained for a given configuration of spheres. Each column of the matrix represents receiver currents obtained for a given transmitting element driven by a unit current.

The imaging methods used here require the complex response matrix for the field scattered back from the spheres. However, in EIGER the calculated response matrix includes mutual coupling between transmit and receive elements. To obtain the contribution from only the scattered field, 
we must remove the mutual coupling component from the total response matrix. This is obtained from simulations performed for each array without the presence of scattering objects. The difference between these results and the simulations with the presence of a scattering object is the contribution from the scattered field alone.

\section{IMAGING METHODS}

The primary imaging method investigated here is a linear subspace method based on MUltiple SIgnal Classification (MUSIC) (Schmidt 1979; Schmidt 1986; Devaney 2002; Lehman et al. 2002; Borcea et al. 2002). In addition, we form images using a more conventional focused beamforming technique (VanTrees 2002) for purposes of comparison. Beamforming is based on a model of the measured scattered field as the sum of fields emitted by a collection of non-interacting point sources. Given an array of $M$ receivers with positions $\mathbf{b}_{m}, m=1,2, \ldots, M$, the received voltages $R_{m}$ are given by

$$
R_{m}=\sum_{l=1}^{L} \sigma_{l} g\left(\mathbf{b}_{m} ; \mathbf{x}_{l}\right)
$$

where $\sigma_{l}$ represent the strengths of the $L$ point sources located at positions labeled $\mathbf{x}_{l}$, and $g(\mathbf{x} ; \mathbf{y})$ is the Green's function, which is assumed known. (This is typically the free space Green's function, but others can be used when appropriate.) A backpropagated field $\psi_{b f}(\mathbf{x})$ is calculated from the received data $R_{m}$ using the assumed Green's function:

$$
\psi_{b f}(\mathbf{x})=\sum_{m=1}^{M} R_{m} g^{*}\left(\mathbf{x} ; \mathbf{b}_{m}\right),
$$

with the point $\mathbf{x}$ varying over a chosen range. An image is obtained by displaying the magnitude of $\psi_{b f}$, or some other quantity derived from $\psi_{b f}$ (e.g. real or imaginary parts). For the case of a scattered field created by point sources, the backpropagated field is obtained by combining equations (1)) and ((2):

$$
\psi_{b f}(\mathbf{x})=\sum_{m=1}^{M} \sum_{l=1}^{L} \sigma_{l} g^{*}\left(\mathbf{x} ; \mathbf{b}_{m}\right) g\left(\mathbf{b}_{m} ; \mathbf{x}_{l}\right) .
$$

Unless $\mathbf{x}=\mathbf{x}_{l}$ for some $l$ (say $l_{1}$ ), the phases of the terms in the sum over $l$ add incoherently and

$\psi_{b f}$ is small. When $\mathbf{x}=\mathbf{x}_{l_{1}}$, phases of terms with $l=l_{1}$ add coherently and $\left|\psi_{b f}\right|$ has a local maximum of approximately

$$
\left|\psi_{b f}\left(\mathbf{x}_{l_{1}}\right)\right| \simeq\left|\sigma_{l_{1}}\right| \sum_{m=1}^{M}\left|g\left(\mathbf{x}_{l_{1}} ; \mathbf{b}_{m}\right)\right|^{2} .
$$

Thus peaks in the magnitude of $\psi_{b f}$ are produced at the positions of the sources. Furthermore, the value of each peak is proportional to the source strength. This motivates the use of focused beamforming for imaging. For extended scatterers, the image from beamforming is expected to produce peaks distributed around the margins of each object.

Focused beamforming can also be considered a specific type of matched field processing (MFP) (Bucker 1976). In this imaging method, the inner products between the receiver data $R$ and a set of matching vectors $P(\mathbf{x})$ generated for a range of positions $\mathbf{x}$ are calculated, $F(\mathbf{x})=P(\mathbf{x}) \cdot R$. An image is typically obtained by displaying the magnitude of the resulting field $F$. The inner 
product can be interpreted as a projection of the data onto the matching vector. The matching vector is typically obtained from a model of the scattering with the expectation that $|F(\mathbf{x})|$ has a peak when $\mathbf{x}$ is the position of a scatterer. By choosing the components of the matching vector to be $P_{m}(\mathbf{x})=g^{*}\left(\mathbf{x}, \mathbf{b}_{m}\right), m=1,2, \ldots, M$, the inner product $F(\mathbf{x})$ is identical to the backpropagated field $\psi_{b f}(\mathbf{x})$. Thus, focused beamforming is a variant of matched field processing with the matching vector generated from the complex conjugate of the Green's function for the medium. Since other choices of matching vector can be made, MFP is a more general form of imaging than beamforming.

Imaging methods based on MUSIC (Schmidt 1979; Schmidt 1986) have been used by a number of researchers to enhance the detection and characterization of scattering objects. They are based on the singular value decomposition (SVD) of the response matrix as described above. In typical applications, the transmit and receive elements of an array are identical and the response matrix is symmetric. However, for our arrays, the transmit and receive elements are separate and the response matrix is no longer symmetric. It will then be useful to develop the MUSIC imaging algorithms for this more general case [see also Lehman and Devaney (Lehman et al. 2002)].

Let $E_{n}, n=1,2, \ldots, N$ represent the $N$ driving currents on the transmit elements of an array, and $R_{m}, m=1,2, \ldots, M$ represent the $M$ receiver currents from the array. These are related through the $M$ by $N$ complex response matrix $\mathbf{K}, R=\mathbf{K} E$. The SVD of $\mathbf{K}$ is given by $\mathbf{K}=\mathbf{U S V}^{\dagger}$, where $\mathbf{U}$ is the matrix of transmitter singular vectors, $\mathbf{V}$ is the matrix of receiver singular vectors, and $\mathbf{S}$ is a diagonal matrix of the singular values $\mu_{l}, l=1,2, \ldots, L$. (The dagger indicates the Hermitian conjugate.) There are $L$ singular values with $L$ transmitter singular vectors $\left(u^{1}, u^{2}, \ldots, u^{L}\right)$ and $L$ receiver singular vectors $\left(v^{1}, v^{2}, \ldots, v^{L}\right)$. Lehman and Devaney (Lehman et al. 2002) showed that for the case of well-separated point scatterers in free space, each singular value represents the apparent strength of one scatterer, and the corresponding singular vectors focus the receiver and transmitter arrays on the scatterer position. In particular, the forward propagated field constructed from the $j$ th transmitter singular vector,

$$
\psi_{j}^{u}(\mathbf{x})=\sum_{n=1}^{N} u_{n}^{j} g\left(\mathbf{x} ; \mathbf{a}_{n}\right),
$$

focuses on the $j$ th scatterer, as well as the backpropagated field constructed from the $j$ th receiver singular vector,

$$
\psi_{j}^{v}(\mathbf{x})=\sum_{m=1}^{M} v_{m}^{j} g^{*}\left(\mathbf{x}, \mathbf{b}_{m}\right),
$$

where $\left\{\mathbf{a}_{n}\right\}$ are the positions of the transmitters and $\left\{\mathbf{b}_{m}\right\}$ are the positions of the receivers.

In the more general case where the scatterers are not necessarily points, the field generated by a selected singular vector may not focus to a single point, but would presumably identify the main contributor(s) to the part of the total scattered field represented by that vector.

The singular values are real and positive and are assumed to be ordered by magnitude, $\mu_{1} \geq \mu_{2} \geq$ $\ldots \geq \mu_{L}$. We use them to divide the space spanned by the singular vectors into a signal subspace and a noise subspace, where the signal subspace is the space spanned by the singular vectors whose singular values are greater than a given threshold. The space spanned by the remaining singular vectors is the noise subspace. The noise threshold is typically chosen by looking for gaps in the spectrum of singular values. It is assumed that a gap indicates that the singular values above the gap are associated with scatterers while those below the gap are associated with noise. The MUSIC 
image for the receiver vectors is constructed from the backpropagated fields of the noise subspace:

$$
\chi_{J}^{v}(\mathbf{x})=\frac{\left|\psi_{0}(\mathbf{x})\right|^{2}}{\sum_{j=J+1}^{L}\left|\psi_{j}^{v}(\mathbf{x})\right|^{2}},
$$

where $\psi_{0}(\mathbf{x})$ is a normalization function, typically unity, and $J$ is the dimension of the signal subspace. The denominator of $\chi_{J}^{v}(\mathbf{x})$ is the length of the projection of the matching vector $P(\mathbf{x})=$ $\left[g\left(\mathbf{x} ; \mathbf{b}_{1}, \ldots, g\left(\mathbf{x} ; \mathbf{b}_{M}\right)\right]^{T}\right.$ onto the noise subspace. This denominator becomes small at values of $\mathbf{x}$ where $P(\mathbf{x})$ is closely aligned with the signal subspace, generating a sharp peak in $\chi_{J}^{v}$. A similar MUSIC image can be generated from the transmitter vectors using the forward propagated fields:

$$
\chi_{J}^{u}(\mathbf{x})=\frac{\left|\psi_{0}(\mathbf{x})\right|^{2}}{\sum_{j=J+1}^{L}\left|\psi_{j}^{u}(\mathbf{x})\right|^{2}} .
$$

The function in the numerator, $\psi_{0}(\mathbf{x})$, is usually taken to be unity (Schmidt 1979; Schmidt 1986; Devaney 2002; Lehman et al. 2002; Borcea et al. 2002). In our case, however, it will be necessary to choose a nontrivial $\psi_{0}$ to reduce the number of spurious peaks in the MUSIC image caused by reflections from the ground.

\section{RESULTS}

\subsection{Single sphere}

We first investigate the simplest case of imaging of a single sphere at the location $\mathbf{x}_{1}=$ $\left(x_{1}, y_{1}, z_{1}\right)=(12.3,-0.75,3.0)$ meters from the origin, illuminated by the linear array (see Fig. 1). As described in section 2, the response matrix is calculated by running EIGER once for each transmitter, retaining the currents on each receiver element. This is done first without a scatterer in order to determine the response matrix caused by mutual coupling between array elements. The procedure is repeated with the scatterer(s) placed in front of the array to determine the total response matrix, including both mutual coupling and scattering. The difference is the response matrix for the scattered field alone. In actual radar systems, mutual coupling is minimized through array design and time gating of the received signal. However, since EIGER is a frequency domain code, we cannot use time gating to exclude mutual coupling from the response matrix. The differencing procedure performs the same function as time gating, allowing us to calculate the response matrix for the scattered field alone. Images of the response matrices for mutual coupling, total field, and the scattered field are shown in Fig. 2. It is immediately apparent that the mutual coupling is significantly stronger than the contribution from the scattered field. The images of the mutual coupling and the total field (Fig. $2 \mathrm{a}$ and $2 \mathrm{~b}$ ) are essentially identical. The scattered field is 3 orders of magnitude smaller than the mutual coupling. Images of the real and imaginary parts of the scattering response matrix (Fig. 2c and 2d) show concentric rings centered around the array element closest to the sphere. This is consistent with a spherical wave originating from the center of the scatterer.

Imaging using MUSIC requires calculating the singular value decomposition (SVD) of the scattering response matrix (see sec. 3). The first four singular values in the spectrum for a single sphere are shown in Fig. 3. The singular values decrease rapidly with the second only $2.4 \%$ of the first, and the third nearly four orders of magnitude below the first. Images obtained by backpropagating the associated receiver singular vectors and evaluating the resulting fields at the height of the sphere (3 meters) are shown in Figs. 4 and 5. The backpropagation operation [Eqn. (6)] requires choosing 
an appropriate Green's function for the imaging geometry. Typically, the Green's function for free space is used,

$$
g(\mathbf{x} ; \mathbf{y})=g_{0}(\mathbf{x} ; \mathbf{y})=\frac{\exp (i k|\mathbf{x}-\mathbf{y}|)}{4 \pi|\mathbf{x}-\mathbf{y}|} .
$$

In our case, however, the presence of the ground reflection can be incorporated into the backpropagation by choosing the ground plane Green's function $g(\mathbf{x} ; \mathbf{y})=g_{1}(\mathbf{x} ; \mathbf{y})=g_{0}(\mathbf{x} ; \mathbf{y})-g_{0}\left(\mathbf{x} ; \mathbf{y}^{\prime}\right)$, where $\mathbf{y}^{\prime}$ is the position of the image point of $\mathbf{y}$ reflected by the ground plane. Since the ground plane is $z=0$ in our configuration, the image point $\mathbf{y}^{\prime}$ of $\mathbf{y}$ is obtained by changing the sign of the $z$ coordinate. It is not obvious which Green's function is the better choice for imaging. To investigate this, we have backpropagated the singular vectors using both the free space Green's function (Fig. 4) and the ground plane Green's function (Fig. 5). In Fig. 4a the first receiver singular vector generates a strong field directed at the sphere (white circle), while the second vector (Fig. 4b) directs a null bordered by two strong lobes toward the sphere. Fields generated by higher order vectors have even less energy directed toward the sphere position (not shown). Introducing the ground reflection (Fig. 5) creates a modulation in range caused by the interference between the direct paths and the reflected paths from the array elements to each field point. This range modulation is determined only by the distance of the array above the ground and is independent of the sphere position.

The MUSIC images of the receiver singular vectors for signal subspaces of dimension $J=1$ and $J=2$ (noise subspace dimensions $L-J=20$ and $L-J=19$ ) using the free space Green's function and normalization $\psi_{0}=1$ are shown in Fig. 6 . These images are obtained by evaluating $\chi_{J}^{v}(\mathbf{x})$ (equation (7)) in the horizontal plane containing the sphere (3 meters above ground). The image for $J=1$ (Fig. 6a) shows the typical 'comet tail' seen in previous images using MUSIC (Devaney 2002; Lehman et al. 2002; Borcea et al. 2002). By adding the second singular value to the signal subspace $(J=2$, Fig. $6 \mathrm{~b})$ the comet tail is much reduced and the peak is close to the center of the sphere (indicated by the black circle). It appears that two singular values are associated with the sphere and must be omitted from the noise subspace to produce good localization. Figure 7 shows MUSIC images using the ground plane Green's function $g_{1}$ that includes the reflections from the ground. These are dominated by peaks that occur at the positions of the nulls in the backpropagated singular vectors (Fig. 5). Only the $J=2$ image has a peak at the sphere position. However, without prior knowledge of the location of the sphere, the MUSIC images using $g_{1}$ could not be used to determine the position of the target, or even the number of targets. As we mentioned previously for the backpropagated fields, the nulls are created by interference between the direct paths and the reflected paths between the array elements and a given field point. This is governed by the geometry of the array and its height above the ground. This pattern also occurs for the field obtained by setting all array elements to unity, i.e. the broadside beam pattern (Fig. 8). If we use this beam pattern to normalize the MUSIC image, that is

$$
\psi_{0}(\mathbf{x})=\sum_{m=1}^{M} g^{*}\left(\mathbf{x}, \mathbf{b}_{m}\right),
$$

the interference effect can be eliminated and the resulting MUSIC images (Fig. 9) are much improved. The $J=2$ image shows only a single peak positioned at the center of the sphere. The Green's function in (10) can be either $g_{0}$ or $g_{1}$ as appropriate. However, normalization by $\psi_{0}$ does not appreciably change MUSIC images obtained from the free space Green's function. Similar 
results are obtained for MUSIC images constructed from the transmitter singular vectors. However, for convenience and purposes of comparison, we only show images obtained from the receiver singular vectors.

We have seen both free space and ground plane Green's functions produce MUSIC images with $J=2$ that when normalized by the broadside beam pattern successfully detects and locates the sphere in the plane containing the array and the sphere. So far it is not clear whether there is any real advantage to using the ground plane Green's function over the free space Green's function. However, we still need to investigate the ability of the MUSIC imaging algorithms to localize the height of the sphere above ground. Fig. 10 shows isosurfaces of the MUSIC functional $\chi_{2}^{v}(\mathbf{x})$ using $g_{0}(10 \mathrm{a})$ and $g_{1}(10 \mathrm{~b})$, both evaluated at $20 \%$ of their respective maxima. It is clear that the MUSIC functional using the free space Green's function has difficulty localizing the height of the sphere. This is consistent with the expected performance of linear arrays in free space. Localization in three dimensions requires a planar array whose aperture is appreciable in two dimensions. However, by incorporating information from the reflected paths, the MUSIC functional using the ground plane Green's function can locate the sphere in all three dimensions with nearly equal precision. The reflected image of the linear array across the ground plane creates a much greater effective vertical aperture that can be exploited by using a Green's function that incorporates the reflected field components.

\subsection{Two spheres}

We now investigate the performance of the MUSIC imaging methods when two spheres are in the field of view (Fig. 11). The second sphere is placed at the position $\mathbf{x}_{2}=\left(x_{2}, y_{2}, z_{2}\right)=(10.4,1.0,3.0)$ meters, which is 2.9 meters from the first sphere in the same horizontal plane. Figure 12 shows the first 6 singular values in the spectrum for this case. In the case of the single sphere, we found that the two largest singular values were associated with the sphere and the rest could be considered noise. Assuming that 2 singular values per sphere is the pattern, we will tentatively identify the first four singular values as being associated with the two spheres (two singular values each). This is equivalent to setting a noise threshold of around $1 \%$ of the largest singular value. It is interesting how the first four singular values seem to be naturally grouped into two pairs, whereas the 5th and 6th eigenvalues appear to be distinct in both magnitude and rate of decrease. This classification is supported by the images of the backpropagated field from the first four receiver vectors shown in Figure 13. The field generated by the vector with the largest singular value is directed toward the closest sphere (Fig. 13a). The second sphere is illuminated by the field associated with the next largest singular vector. The third and fourth singular vectors direct nulls toward the two spheres, surrounded by areas of large field magnitude. Backpropagated fields created by higher order singular vectors send energy in directions further away from both spheres (not shown).

We calculate MUSIC images using the ground plane Green's function with normalization by the broadside beam pattern. Figure 14 shows images for signal subspace dimensions 1 through 5 . The best image is obtained for $J=4$, which is consistent with a noise threshold between singular values 4 and 5. If we lower the threshold to a position between singular values 5 and 6 , we obtain Fig. $14 \mathrm{e}$, which is noticeably worse than Fig. $14 \mathrm{~d}(J=4)$. Three dimensional images using the MUSIC functional for both the free space and ground plane Green's functions are shown in Figure 15. Again, the ground plane Green's function localizes the spheres in height better than the free space Green's function. However, we do see some periodicity in the z direction for Fig. 15b. Apparently only two samples in the $\mathrm{z}$ direction (the array and its reflected image) are not sufficient to eliminate all height ambiguities. 
We have seen that the MUSIC imaging approach works well for localizing one and two spheres with a linear array. The best performance is obtained when two singular values are associated with each sphere, which is consistent with a noise threshold of $1 \%$ of the largest singular value. The MUSIC image is calculated using the ground plane Green's function, which incorporates the effect of reflections from the ground, combined with normalization by the broadside beam pattern. Incorporating the reflected energy significantly improves the height localization but does not completely eliminate ambiguity in height as seen in the results for the two spheres (Fig. 15b). These preliminary conclusions will be tested by attempting to image three spheres.

\subsection{Three spheres - case 1}

Figure 16 shows a configuration of three spheres spaced between 2.5 to 3.3 meters apart. Spheres 1 and 3 are at the same height as the array while sphere 2 is $60 \mathrm{~cm}$ below the plane. Sphere 3 is directly behind sphere 1, measured along a line from the array center to sphere 1 , so that it is obscured in the direct line from the array but not from the array image in the ground plane. The singular value spectrum in Fig. 17 shows 5 singular values above the $1 \%$ threshold instead of 6 expected ( 2 per sphere). If there is a sixth singular value associated with the third sphere, it apparently is too small to rise above the noise threshold. The backpropagated fields for the first 5 receiver vectors shown in Fig. 18 are clearly associated with one or more spheres. Fields for vectors 1 and 2 show maximim intensity along lines directed through the spheres. The fields for vectors 3,4 , and 5 have nulls directed toward the spheres, with maximum intensity between the spheres (vector 3) or on either side (vectors 4 and 5). It is less clear whether the field for the 6th vector (Fig. 18f) is associated with any given sphere. The areas of maximum intensity are directed away from the sector that contains the spheres. The fields are evalulated at the height of the array $(3$ meters), which is $60 \mathrm{~cm}$ above sphere 2 . However, the influence of sphere 2 is clearly visible in the fields for vectors 1, 4, and 5, even above the plane of sphere 2. Evaluating the fields in the horizontal plane that includes sphere 2 gives essentially the same results.

A sequence of MUSIC images for increasing dimensions of the signal subspace are shown in Figure 19. The localization performance improves as the signal subspace dimension $J$ increases from 1 to 5. However, for signal subspace dimension of 6 (Fig. 19f), extra peaks in the image appear even though there appears to be an improvement in the localization of sphere 3 behind sphere 1. Figure 20 shows the $10 \%$ isosurfaces for $J=4,5$, and 6 . Long tubular structures greatly increase in number for $J=6$, suggesting an optimal noise threshold of $1 \%$ that includes $J=5$ but not $J=6$.

\subsection{Three spheres - case 2}

A second configuration where sphere 3 is placed 7.4 meters behind sphere 1 (whereas it was 3 meters behind previously) is analyzed to determined which singular values were most influenced by sphere 3 (see Fig, 21). Comparing the spectrum of singular values (Fig. 22) with the previous spectrum (Fig. 18) shows that singular values 2, 3, and 4, are most affected by the change in sphere 3. Singular value 2 decreases while singular values 3 and 4 increase. There is little or no change in the other singular values.

The backpropagated fields from the receiver singular vectors (Fig. 23) look similar to those for the previous configuration (Fig. 18), with the most differences seen in the fields from the 4th and 5 th vectors. The field from the 4 th vector is directed more toward sphere 1 in the second sphere configuration while the field from 5 th singular vector is directed more toward sphere 2 . As expected, the influence of sphere 3 is less when positioned at a greater distance from the array. 
Figure 24 shows a sequence of MUSIC images equivalent to those in Fig. 19. The greatest difference is seen for a signal subspace dimension of $J=4$. The third sphere is poorly located in all of the images. Poor localization is also seen in the isosurfaces shown in Fig. 25 for $J=4,5$, and 6 . Overall the performance of MUSIC is less satisfactory in the presence of three spheres compared with the earlier one and two sphere configurations. We see a degradation of height localization as well as an inability to determine the range of the sphere located behind the first sphere. This may reflect a limit in the ability of a linear array to estimate range and height. Better performance may be possible with a planar array that would have both length and height. We consider this possibility next.

\subsection{Three spheres - planar array}

We tested the performance of a planar array using the 21 element planar array described in section 2 to image the first configuration of three spheres (Fig. 26). The first 7 singular values in the spectrum appear naturally grouped into 3 sets (Fig. 27). This is reflected in the backpropagated fields generated from the singular vectors (Fig. 28). The first two singular vectors generate fields that direct maximum intensity along the lines defined by the center of the array and the locations of each sphere. Singular vectors 3 and 4 direct intensity along one or the other line of spheres, while vectors 5, 6, and 7 direct nulls toward the spheres. The MUSIC images for $\mathrm{J}=1$ through 8 evaluated at a height of 3 meters are shown in Fig. 29. As J increases, the second sphere lying at $60 \mathrm{~cm}$ below the plane gradually disappears. The isosurfaces shown in Fig. 30 show maxima of the MUSIC functional near the center of sphere 2 but these are more localized at the sphere height than the previous case with the linear array. The planar array is clearly superior to the horizontal linear array in locating the height of the spheres.

Overall the results from imaging configurations of 3 spheres are less satisfactory than the images for 1 or 2 spheres. There appears to be a greater sensitivity of the imaging performance with sphere configuration. If one sphere is signficantly farther from the array than the others, it is difficult to localize by simply thresholding the MUSIC functional without introducing false targets. Also, in both cases, the third sphere is shadowed by one of the other spheres and localization is more difficult. Performance is improved if the physical array is planar rather than linear, with better height localization. Certainly the MUSIC functional contains information on all the spheres but there is also room for improvement in extracting that information. Further investigation of the connection between the singular values of the response matrix and the targets is expected to provide better criteria for target identification.

\section{SUMMARY AND CONCLUSIONS}

We have shown that multiple spheres can be imaged by linear and planar EM arrays using only one component of polarization. The imaging approach involves calculating the SVD of the scattering response matrix, selecting a subset of singular values that represents noise, and evaluating the MUSIC functional. The noise threshold applied to the spectrum of singular values for optimal performance is typically around $1 \%$. The resulting signal subspace includes more than one singular value per sphere. The presence of reflections from the ground improves height localization, even for a linear array parallel to the ground. However, the interference between direct and reflected energy modulates the field, creating periodic nulls that can obscure targets in typical images. These nulls are largely eliminated by normalizing the MUSIC functional with the broadside beam pattern of the array. The resulting images show excellent localization for 1 and 2 spheres. The performance for the 3 sphere configurations are complicated by shadowing effects and the greater range of the 3rd 
sphere in case 2. Two of the three spheres are easily located by MUSIC but the third is difficult to distinguish from other local maxima of the complex imaging functional. Improvement is seen when the linear array is replace with a planar array, which increases the effective aperture height. Further analysis of the singular values and their relationship to modes of scattering from the spheres, as well as better ways to exploit polarization, should improve performance. Work along these lines is currently being pursued by the authors.

\section{ACKNOWLEDGMENTS}

This work was performed under the auspices of the U. S. Department of Energy by the University of California, Lawrence Livermore National Laboratory under Contract No. W-7405-Eng-48.

\section{REFERENCES}

Berryman, J. G., Borcea, L., Papanicolaou, G. C., and Tsogka, C. (2002). "Statistically stable ultrasonic imaging in random media." J. Acoust. Soc. Am., 112, 1509-1522.

Borcea, L., Papanicolaou, G. C., Tsogka, C., and Berryman, J. G. (2002). "Imaging and time reversal in random media." Inverse Problems, 18, 1247-1279.

Bucker, H. P. (1976). "Use of calculated sound field and matched-field detection to locate sound sources in shallow water." J. Acoust. Soc. Am., 59, 368-373.

Chambers, D. H. and Gautesen, A. K. (2001). "Time reversal for a single spherical scatterer." $J$. Acoust. Soc. Am., 109, 2616-2624.

Champagne II, N. J., Berryman, J. G., and Buettner, H. M. (2001). "FDFD: A 3D finite-difference frequency-domain code for electromagnetic induction tomography." J. Comput. Phys., 170, 830848.

Claerbout, J. F. (1976). Fundamentals of Geophysical Data Processing with Applications to Petroleum Prospecting. McGraw-Hill, New York.

Devaney, A. J. (2002). "Super-resolution processing of multi-static data using time reversal and MUSIC. to appear in JASA.

Durden, S. L., Klein, J. D., and Zebker, H. A. (1991). "Radar measurement of L-band signal fluctuations caused by propagation through trees." IEEE Trans. Antennas Propag., 39, 15371539.

Durden, S. L., Klein, J. D., and Zebker, H. A. (1993). "Measurement and simulation of signal fluctuations caused by propagation through trees." Radio Science, 28, 1049-1051.

Fink, M. (March, 1997). "Time reversal acoustics." Physics Today, 50, 34-40.

Fink, M. (November, 1999). "Time-reversed acoustics." Scientific American, 281, 91-97.

Fink, M. and Prada, C. (2001). "Acoustic time-reversal mirrors." Inverse Problems, 17, R1-R38.

Fleischman, J. G., Ayasli, S., Adams, E. M., and Gosselin, D. R. (1996). "Foliage penetration experiment Part I: Foliage attenuation and backscatter analysis of SAR." IEEE Trans. Aerospace Electronic Sys., 32, 135-144.

Fleischman, J. G., Worris, M. A., Ayasli, S., Adams, E. M., and Gosselin, D. R. (1996). "Foliage penetration experiment Part III: Multichannel whitening of SAR imagery." IEEE Trans. Aerospace Electronic Sys., 32, 156-166.

Karam, M. A., Fung, A. K., Lang, R. H., and Chauhan, N. S. (1992). "A microwave scattering model for layered vegetation." IEEE Trans. Geosci. Remote Sensing, 30, 767-784.

Lehman, S. K., Devaney, A. J., and Witten, A. J. (2002). "Geophysical subsurface acoustic timereversal imaging. 
Prada, C. and Fink, M. (1994). "Eigenmodes of the time reversal operator: A solution to selective focusing in multiple-target media." Wave Motion, 20, 151-163.

Prada, C., Manneville, S., Spoliansky, D., and Fink, M. (1996). "Decomposition of the time reversal operator: Detection and selectiv e focusing on two scatterers." J. Acoust. Soc. Am., 99, 20672076.

Prada, C., Thomas, J.-L., and Fink, M. (1995). "The iterative time-reversal process: Analysis of the convergence." J. Acoust. Soc. Am., 97, 62-71.

Prada, C., Wu, F., and Fink, M. (1991). "The iterative time reversal mirror: A solution to selffocusing in the pulse echo mode." J. Acoust. Soc. Am., 90, 1119-1129.

Sarabandi, K. and Koh, I.-S. (2001). "A complete physics-based channel parameter simulation for wave propagation in a forest environment." IEEE Trans. Antennas Propag., 49, 260-271.

Schmidt, R. O. (1979). "Multiple emitter location and signal parameter estimation." Proc. RADC Spectrum Estimation Workshop, Rome Air Development Center, Rome, NY. 243-258. RADCTR-79-63.

Schmidt, R. O. (1986). "Multiple emitter location and signal parameter estimation." IEEE Trans. Antennas Propag., AP-34, 276-281.

Senior, T. B. A., Sarabandi, K., and Ulaby, F. T. (1987). "Measuring and modeling the backscattering cross section of a leaf." Radio Science, 22, 1109-1116.

ter Haar, G. (December, 2001). "Acoustic surgery." Physics Today, 54, 29-34.

Theron, I. P., Walton, E. K., Gunawan, S., and Cai, L. (1999). "Ultrawide-band noise radar in the VHF/UHF band." IEEE Trans. Antennas Propag., 47, 1080-1084.

Thomas, J.-L., Wu, F., and Fink, M. A. (1996). "Time reversal focusing applied to lithotripsy." Ultrasonics Imaging, 18, 106-121.

Toups, M. F., Ayasli, S., and Fleischman, J. G. (1996). "Foliage penetration experiment Part II: Analysis of foliage-induced synthetic pattern distortions." IEEE Trans. Aerospace Electronic Sys., 32, 145-155.

Ulaby, F. T., Sarabandi, K., McDonald, K., Whitt, M., and Dobson, M. C. (1990). "Michigan microwave canopy scattering model." Intern. J. Remote Sensing, 11, 1223-1253.

VanTrees, H. L. (2002). Optimum Array Processing. Wiley, New York.

White, D. A., Sharpe, R., and Champagne, N. (2000). "Solution of hybrid FEM-BEM systems via Schur complement techniques. LLNL UCRL-ID-141669.

$\mathrm{Xu}, \mathrm{X}$. and Narayanan, R. M. (2001a). "FOPEN SAR imaging using UWB step-frequency and random noise waveforms." IEEE Trans. Aerospace Electronic Sys., 37, 1287-1300.

$\mathrm{Xu}, \mathrm{X}$. and Narayanan, R. M. (2001b). "Range sidelobe suppression technique for coherent ultra wide-band random noise radar imaging." IEEE Trans. Antennas Propag., 49, 1836-1842. 


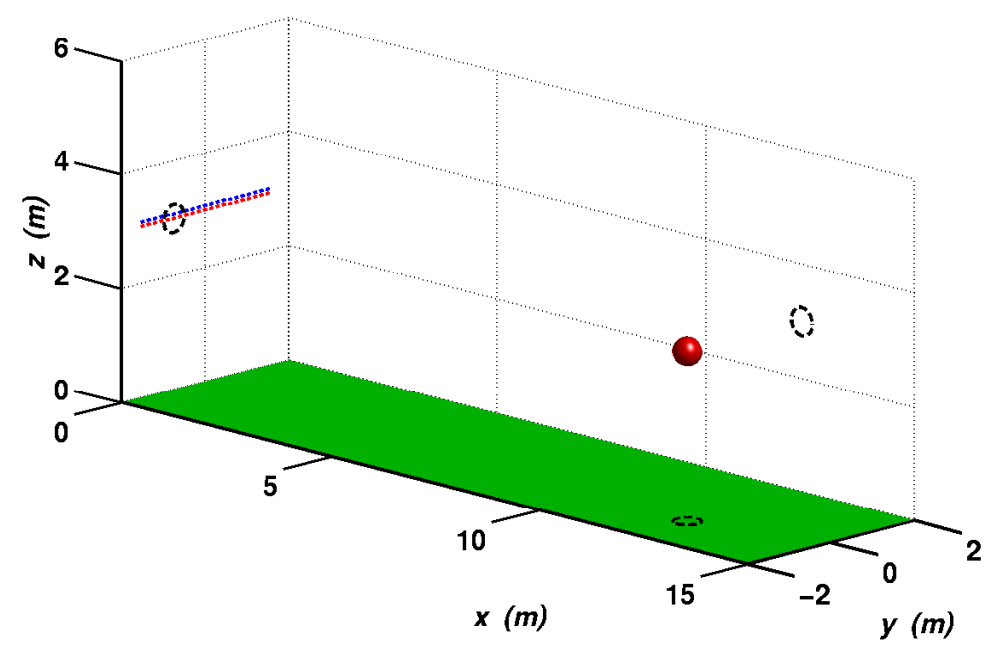

FIG. 1. Geometry of single sphere simulation. The linear array in the yz plane is composed of 21 transmit elements (red) and 21 receiver elements (blue). The $60 \mathrm{~cm}$ diameter sphere is centered at the point $(12.3,-.75,3.0)$ meters.
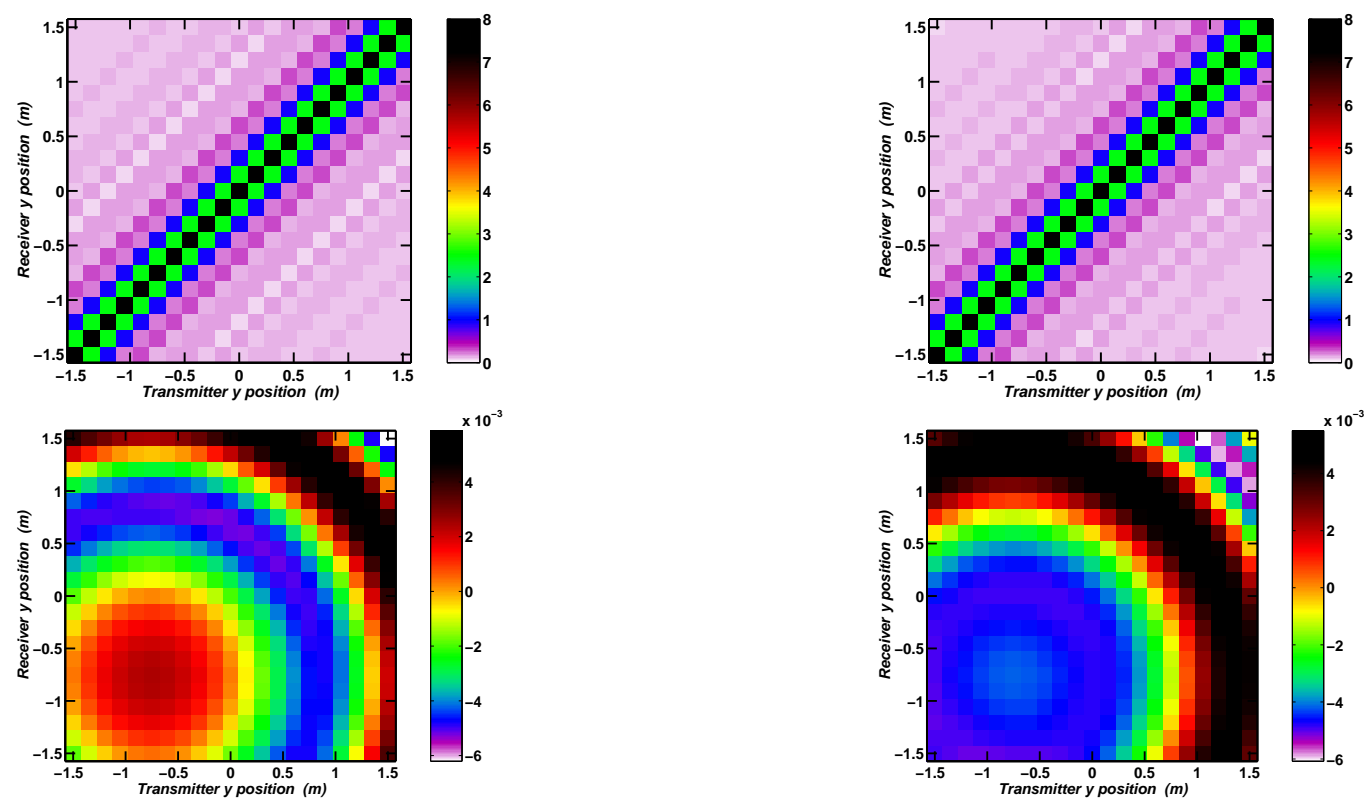

FIG. 2. Magnitude of the response matrix without a sphere (mutual coupling only) (a), and with a single sphere (total field) (b). Real (c) and imaginary (d) parts of the response matrix for the scattered field from a single sphere. 


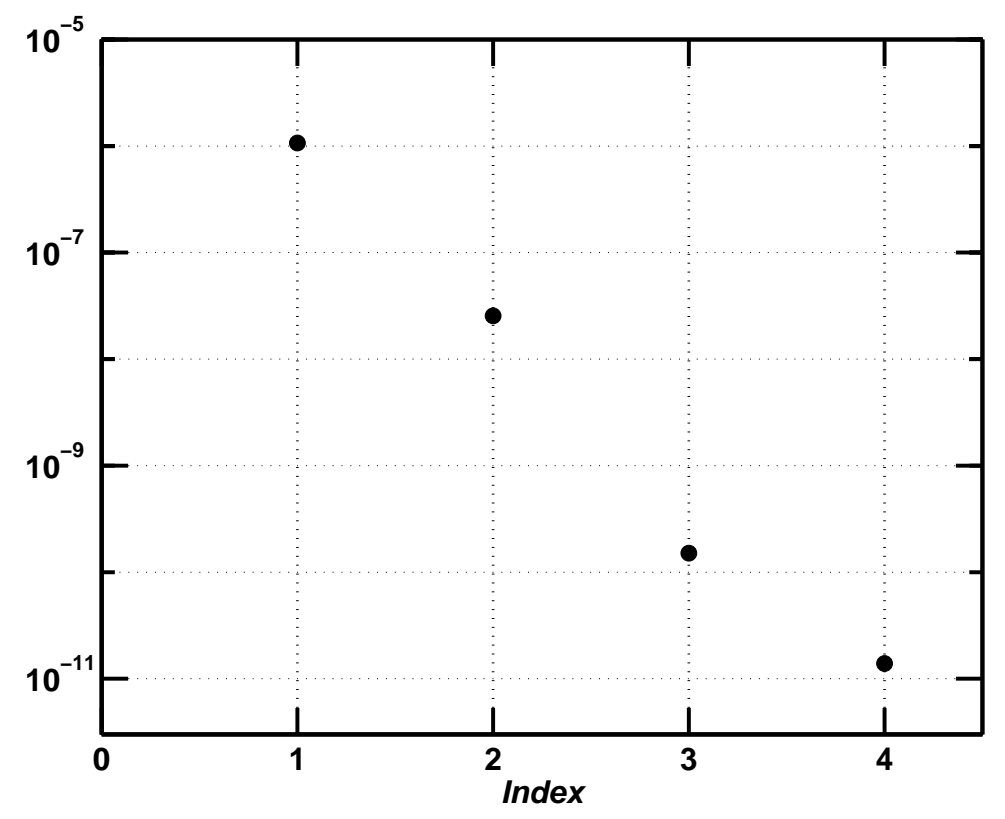

FIG. 3. First four singular values in the spectrum for a single sphere.
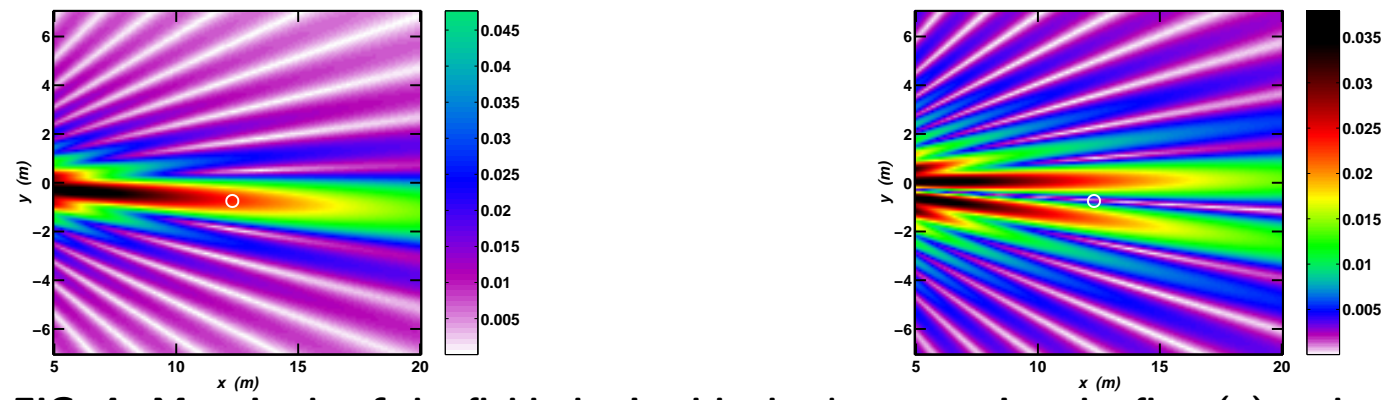

FIG. 4. Magnitude of the field obtained by backpropagating the first (a) and second (b) singular vectors using the free space Greens function. The field is evaluated in the plane of the sphere at a height of 3 meters. The intersection of the sphere with the image plane is indicated by the white circle. 

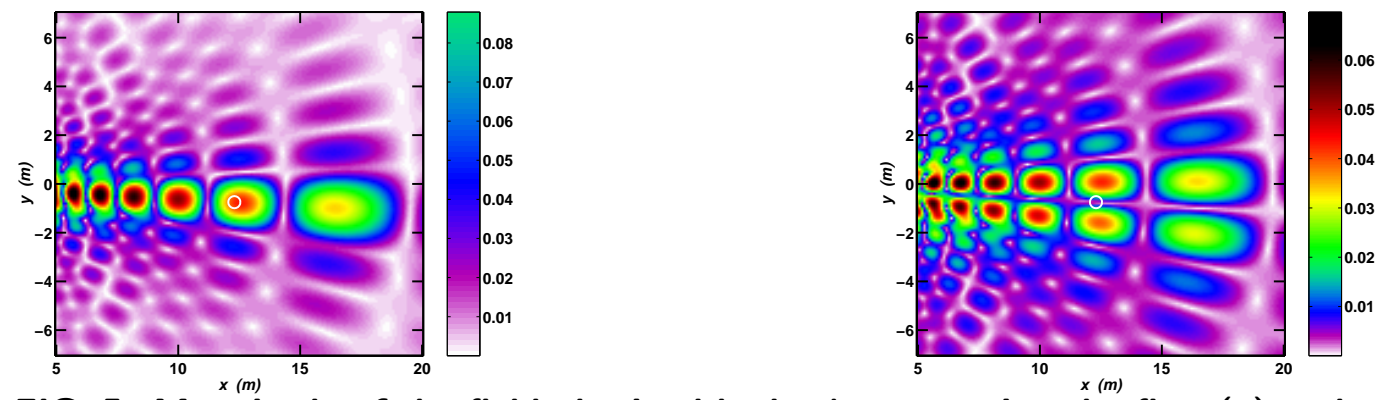

FIG. 5. Magnitude of the field obtained by backpropagating the first (a) and second (b) singular vectors using the Greens function that includes the ground reflections. The field is evaluated in the plane of the sphere at a height of 3 meters. The intersection of the sphere with the image plane is indicated by the white circle.
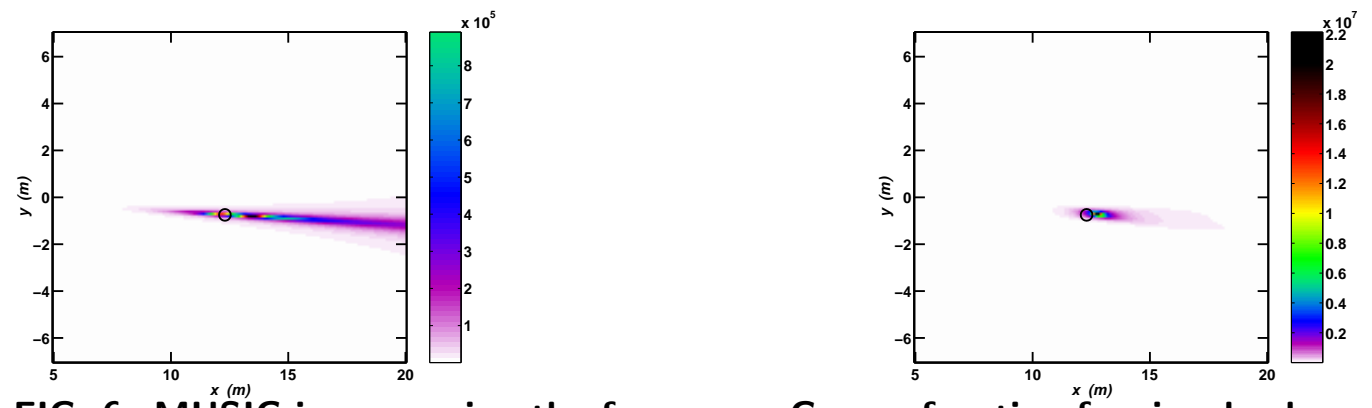

FIG. 6. MU SIC images using the free space Greens function for signal subspace dimensions of 1 (a) and 2 (b) evaluated in the plane of the sphere (black circle) at a height of 3 meters.
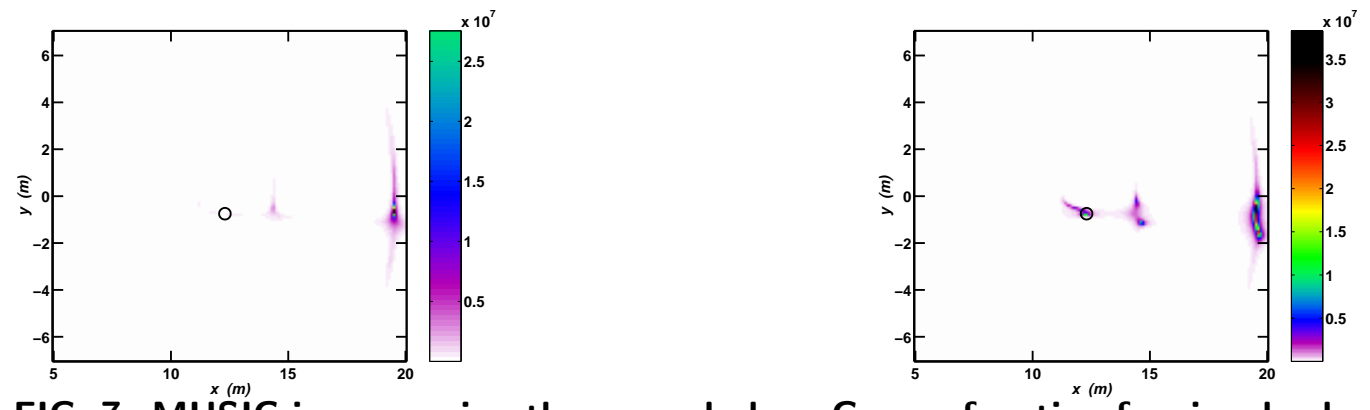

FIG. 7. MUS' IC images using the ground plane Greens function for signal subspace dimensions of 1 (a) and 2 (b) evaluated in the plane of the sphere (black circle) at a height of 3 meters. These images have not been normalized by the broad side beam pattern. 


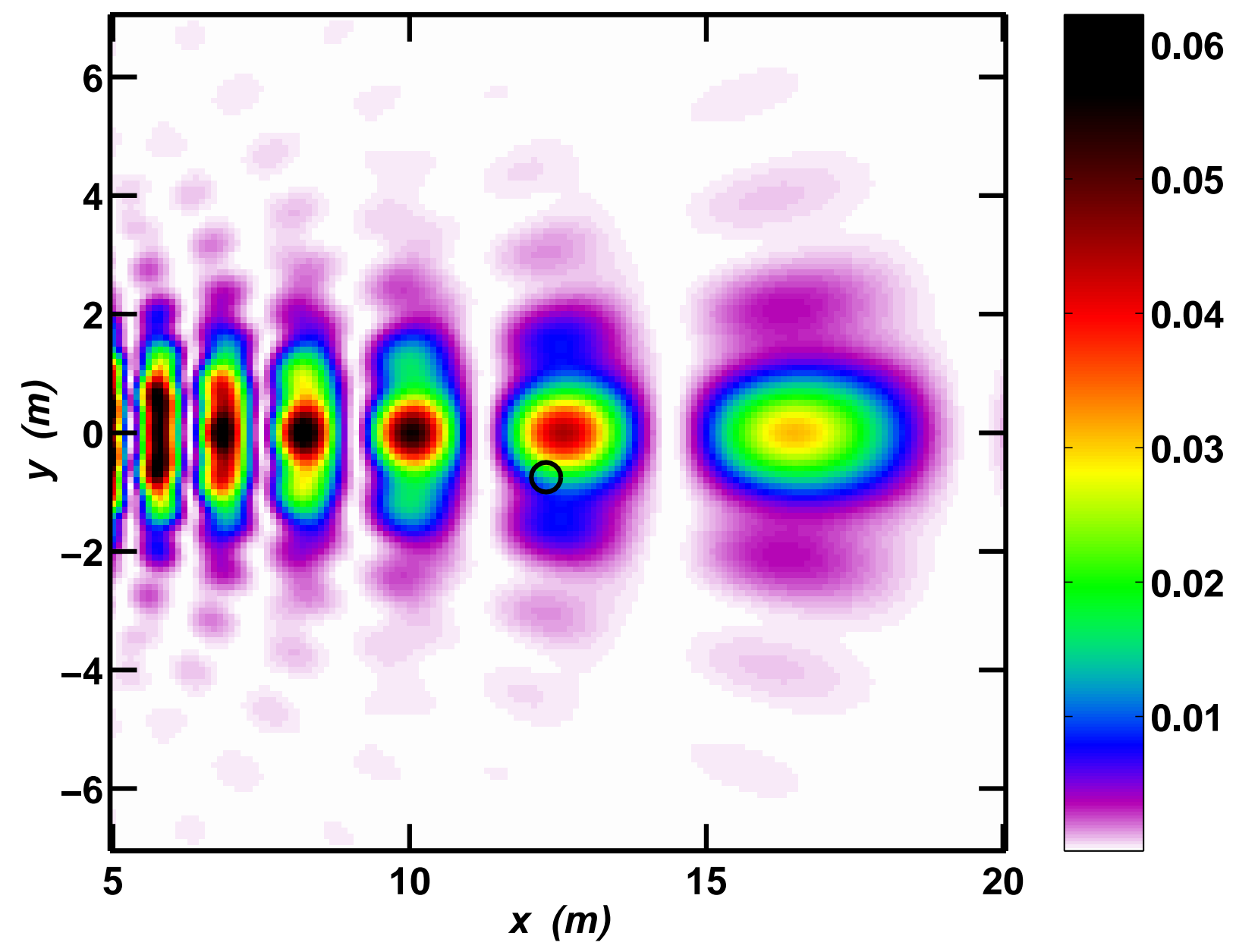

FIG. 8. Magnitude of the backpropagated field generated by transmitting unit amplitude on all array elements (broadside beam pattern). Field evaluated in the plane of the sphere (black circle) at a height of 3 meters.
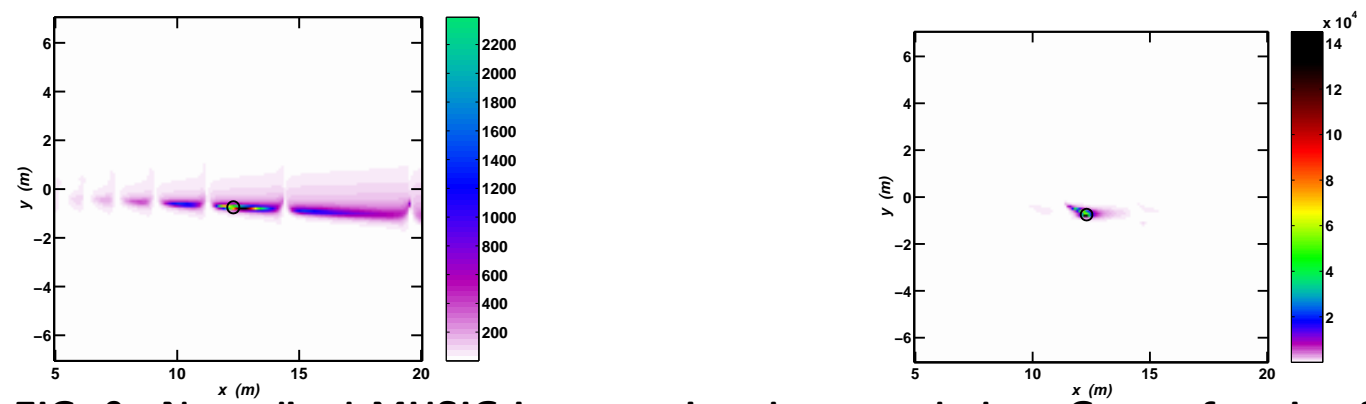

FIG. 9. Normalized MUSIC images using the ground plane Greens function for signal subspace dimensions of 1 (a) and 2 (b) evaluated in the plane of the sphere (black circle) at a height of 3 meters. 

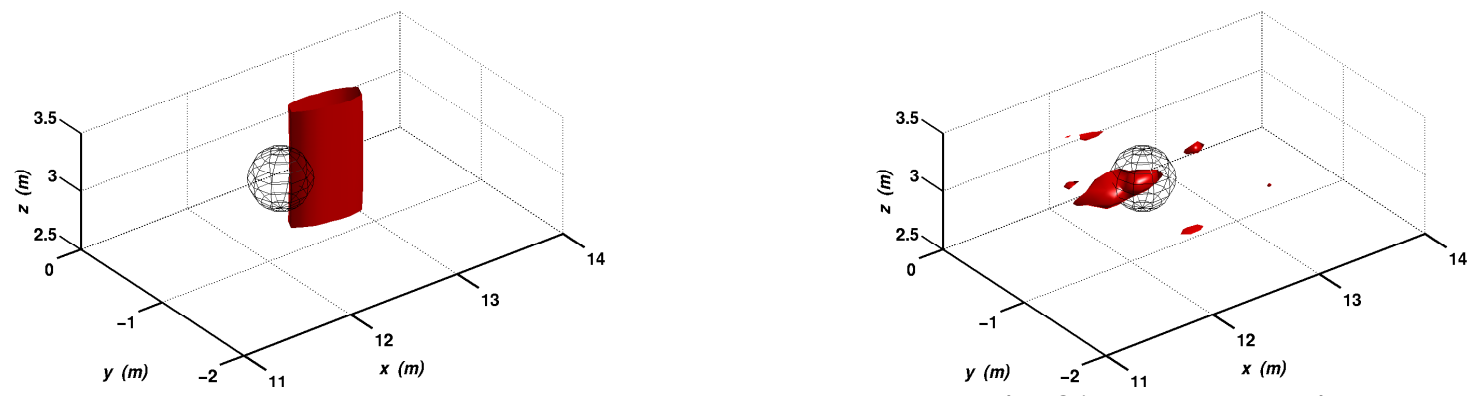

FIG. 10. Isosurfaces of the normalized MUSIC functional (20\% of maximum) using the free space Greens function (a) and ground plane Greens function (b). Sphere is shown as an open mesh. Incorporating information from ground reflections greatly enhances height localization.

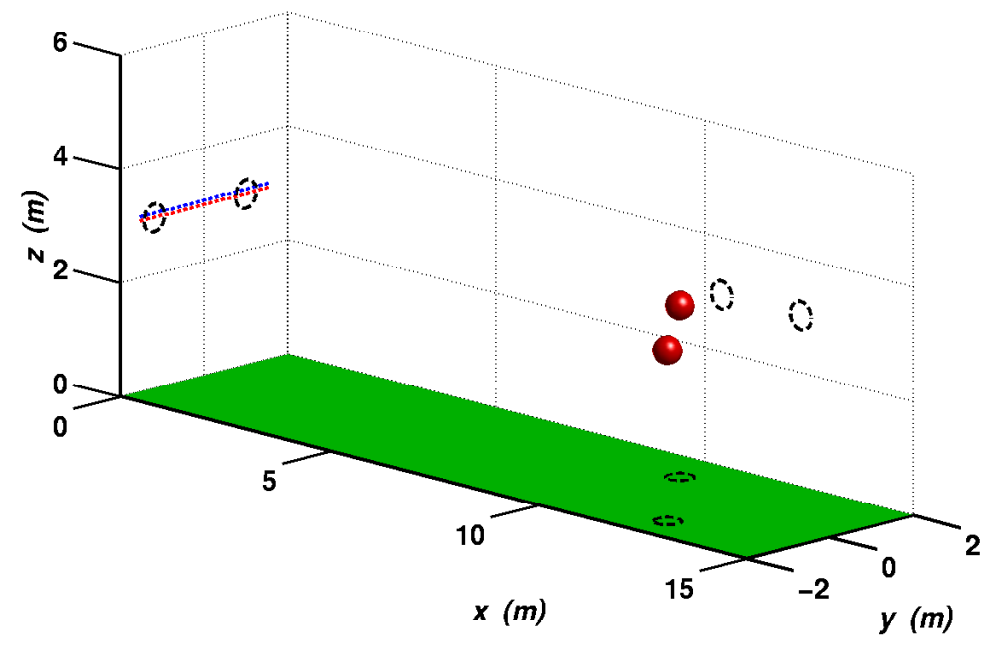

FIG. 11. Geometry of two spheres simulation. The linear array in the yz plane is composed of 21 transmit elements (red) and 21 receiver elements (blue). The $60 \mathrm{~cm}$ diameter spheres are centered at the points $(12.3-1.23)$ and $(10.4,1.0,3.0)$ meters. 


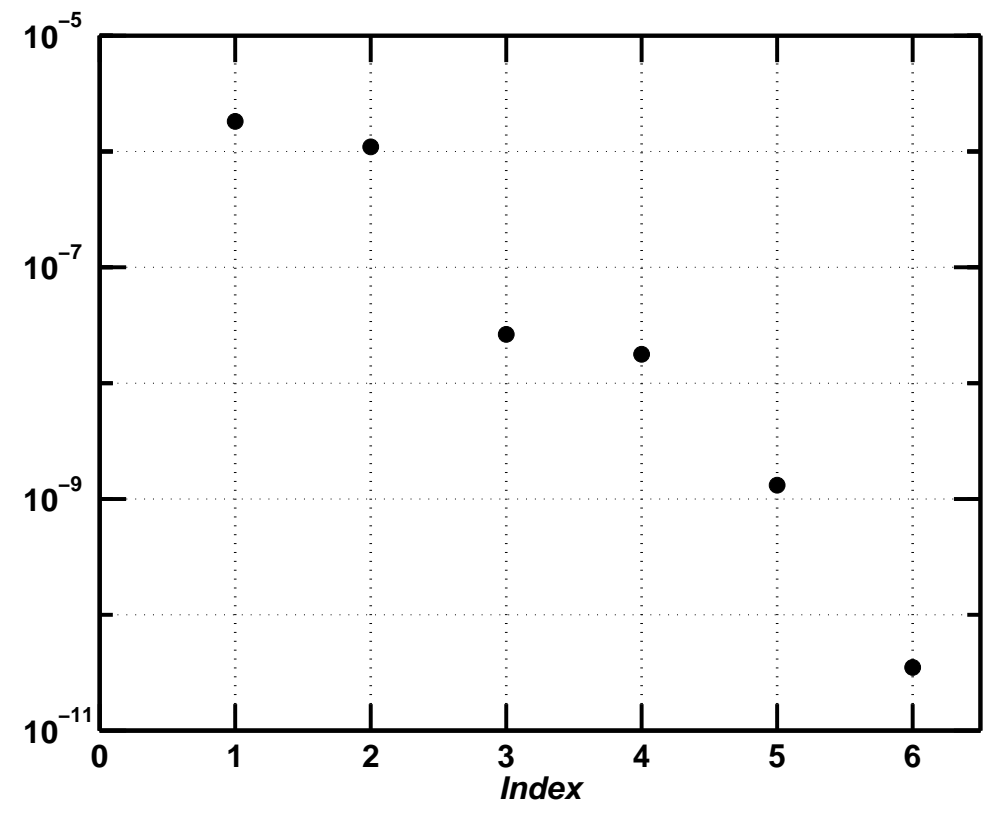

FIG. 12. First six singular values in the spectrum for two spheres.
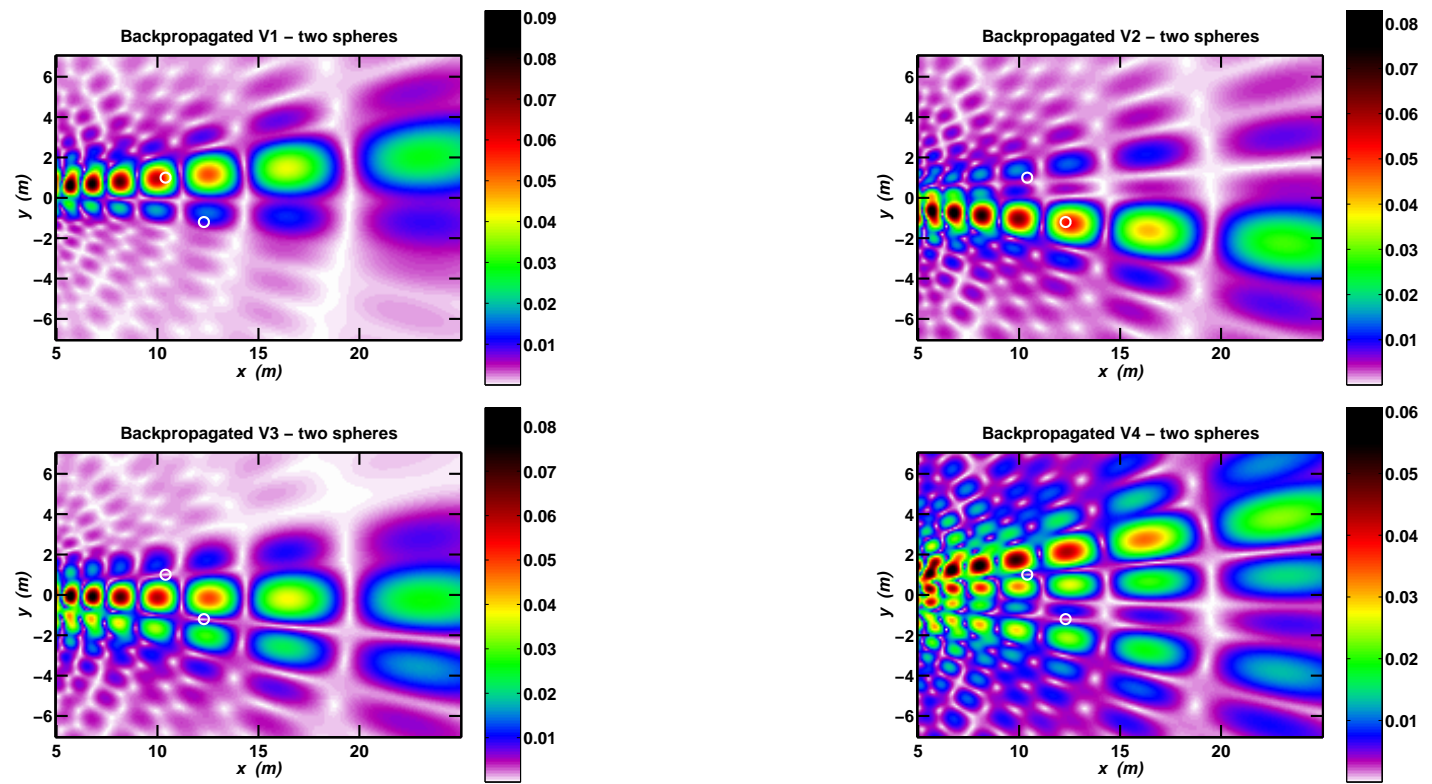

FIG. 13. Magnitude of the fields obtained by backpropagating the first (a), second (b), third (c), and fourth (c) singular vectors using the ground plane Greens function. The field is evaluated in the plane of the spheres at a height of 3 meters. The intersection of the spheres with the image plane is indicated by the white circle. 

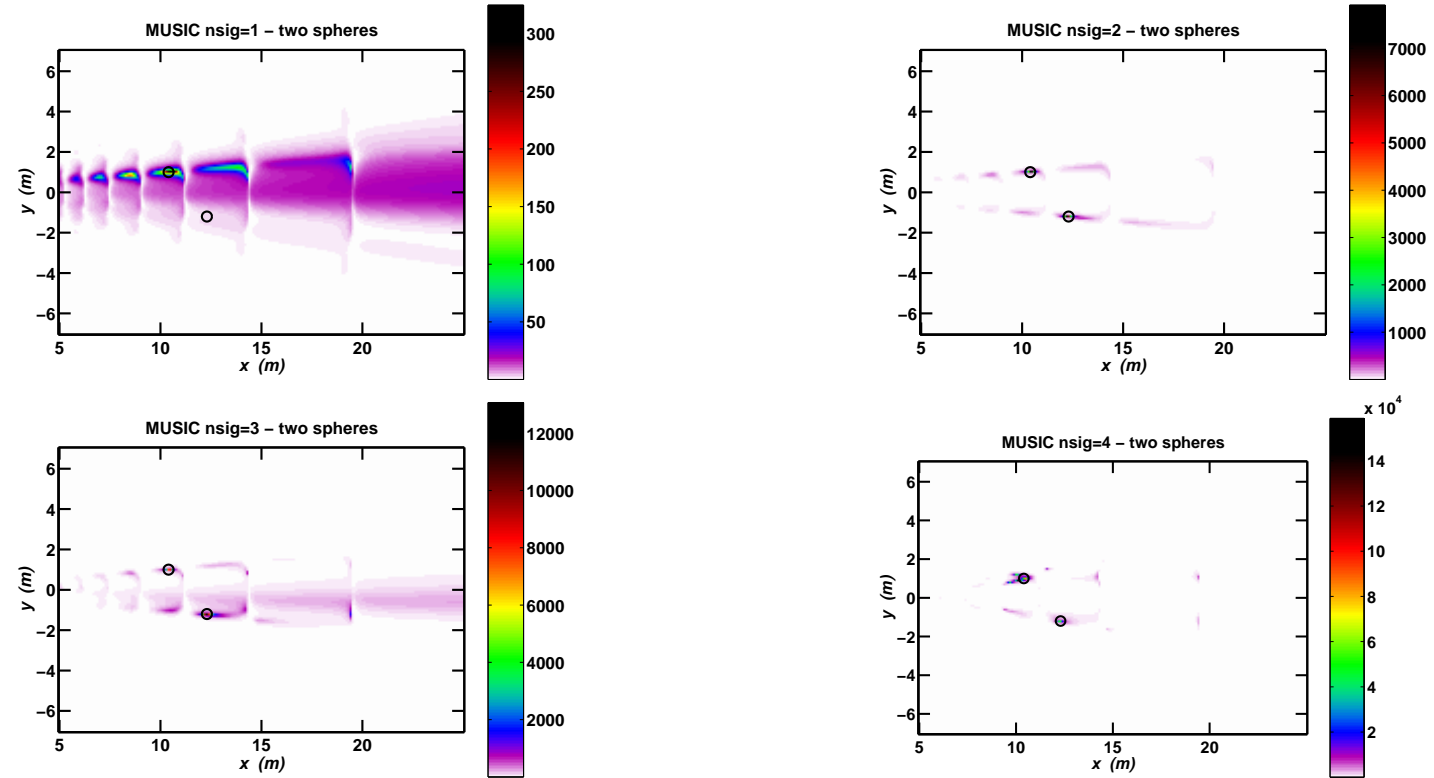

FIG. 14. Normalized MUSIC images using the ground plane Greens function for signal subspace dimensions of 1 (a), 2 (b), 3 (c), and 4 (d) evaluated in the plane of the spheres (black circles) at a height of 3 meters.
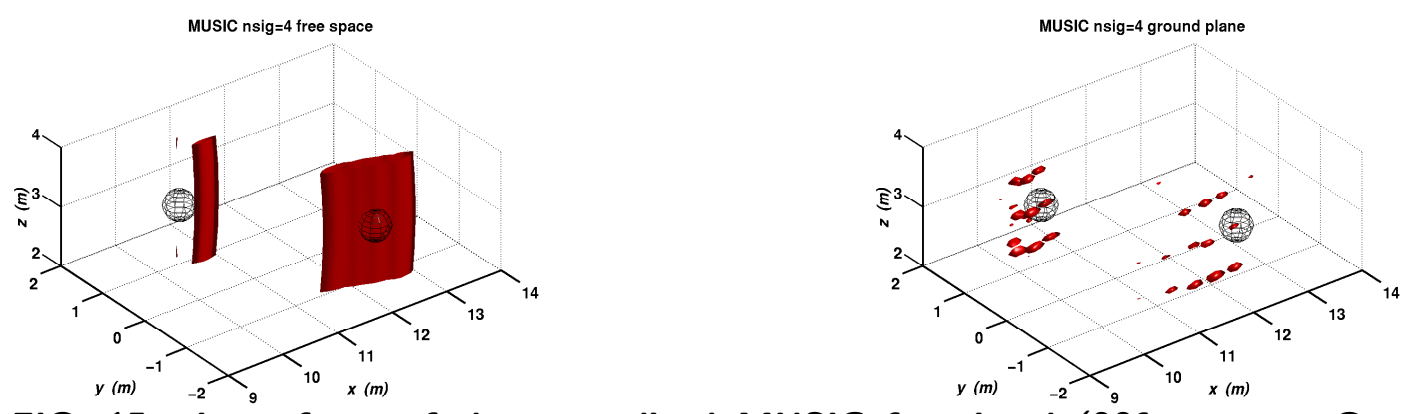

FIG. 15. Isosurfaces of the normalized MUSIC functional (20free space Greens function (a) and ground plane Greens function (b). Spheres are shown as an open meshes. Incorporating information from ground reflections greatly enhances height localization. 


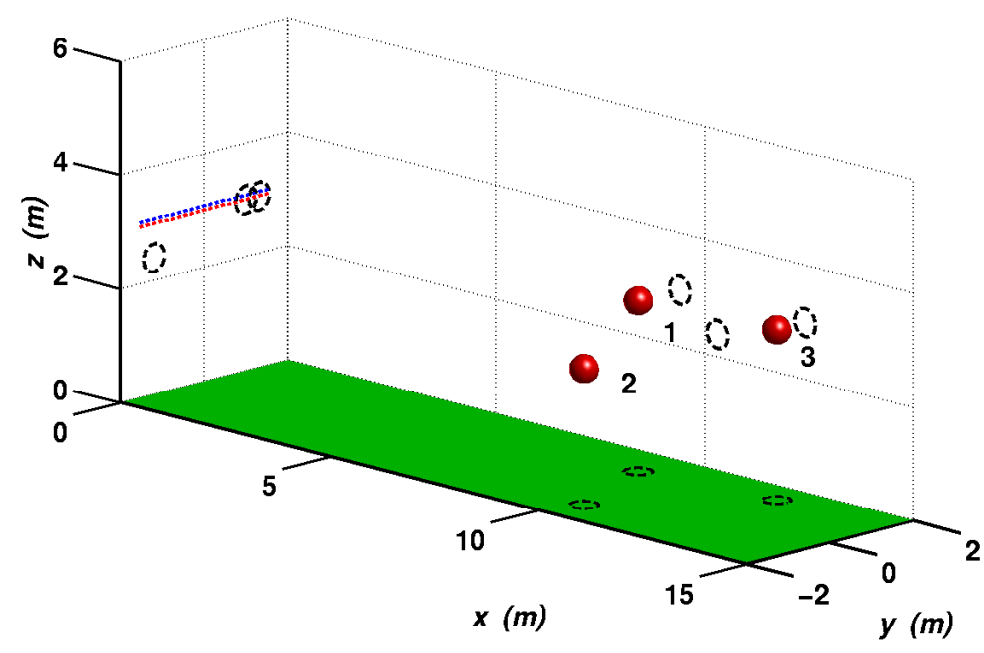

FIG. 16. Geometry of three spheres simulation - case 1 . The linear array in the yz plane is composed of 21 transmit elements (red) and 21 receiver elements (blue). The $60 \mathrm{~cm}$ diameter spheres are centered at the points $(10.3-1.22 .4),(9.4,1.0,3.0)$, and $(12.4,1.32,3.0)$ meters.

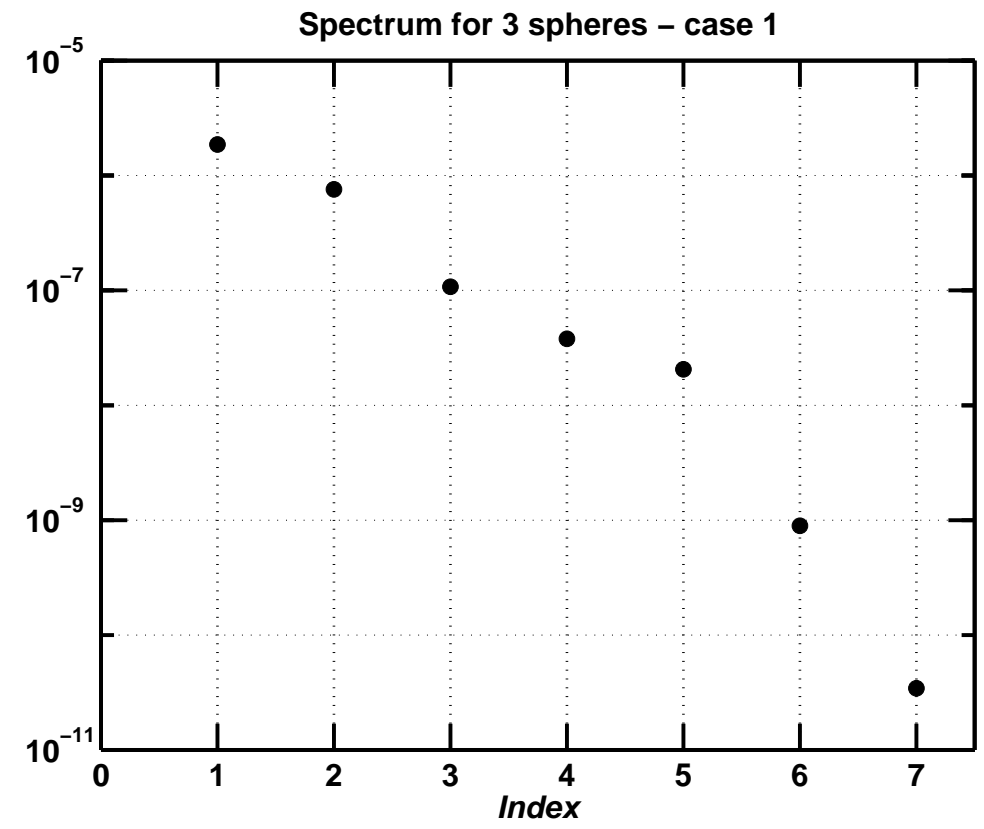

FIG. 17. First seven singular values in the spectrum for three spheres - case 1. 

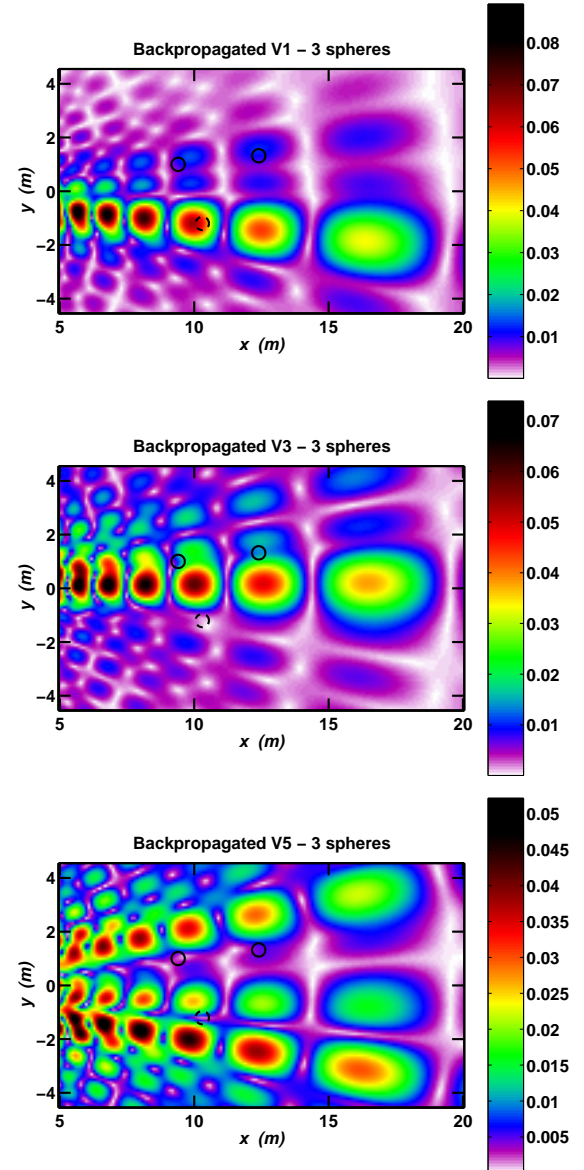
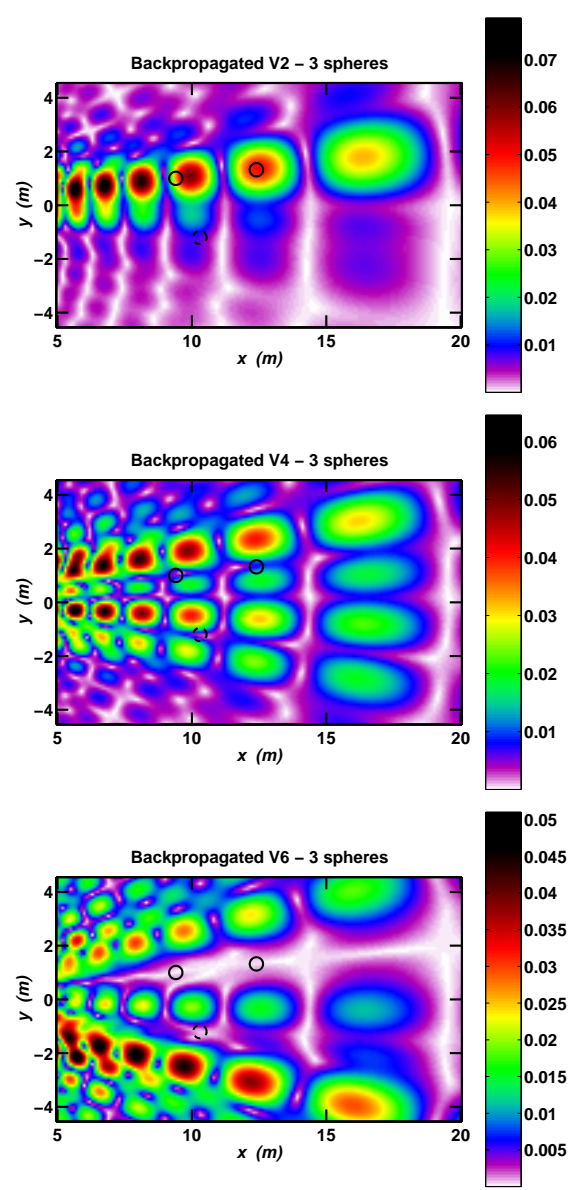

FIG. 18. Magnitude of the fields obtained by backpropagating the first (a), second (b), third (c), fourth (d), fifth (e), and sixth (f) singular vectors using the ground plane Greens function (case 1). The field is evaluated in the plane 3 meters above the ground. The intersections of the two spheres in the image plane are shown as black circles. The third sphere is centered $60 \mathrm{~cm}$ below the dashed circle in the image plane. 

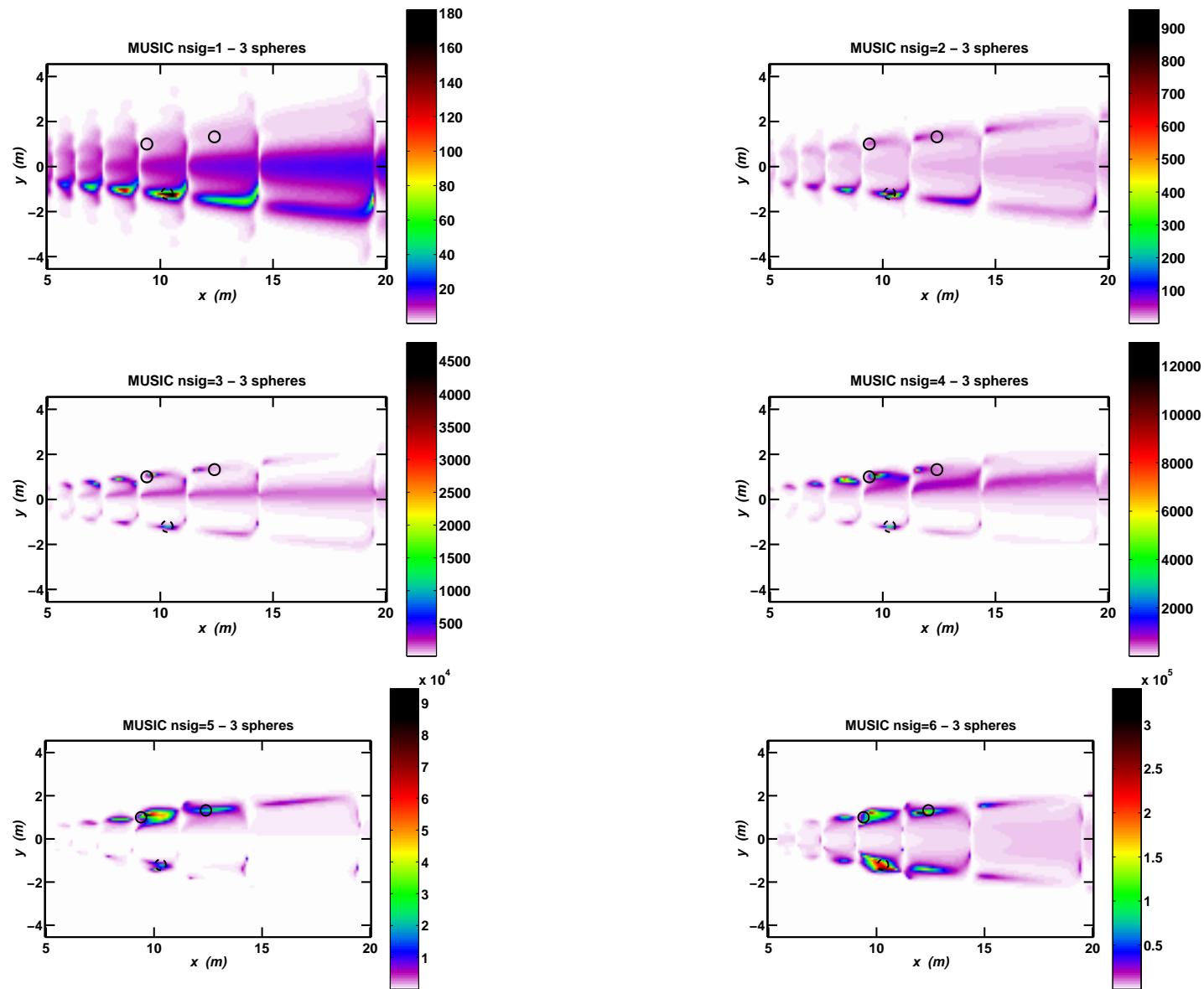

FIG. 19. Normalized MUSIC images using the ground plane Greens function for signal subspace dimensions of 1 (a), 2 (b), 3 (c), 4 (d), 5 (e), and 6 (f) evaluated in the plane 3 meters above the ground (case 1). The intersections of the two spheres in the image plane are shown as black circles. The third sphere is centered $60 \mathrm{~cm}$ below the dashed circle in the image plane. 

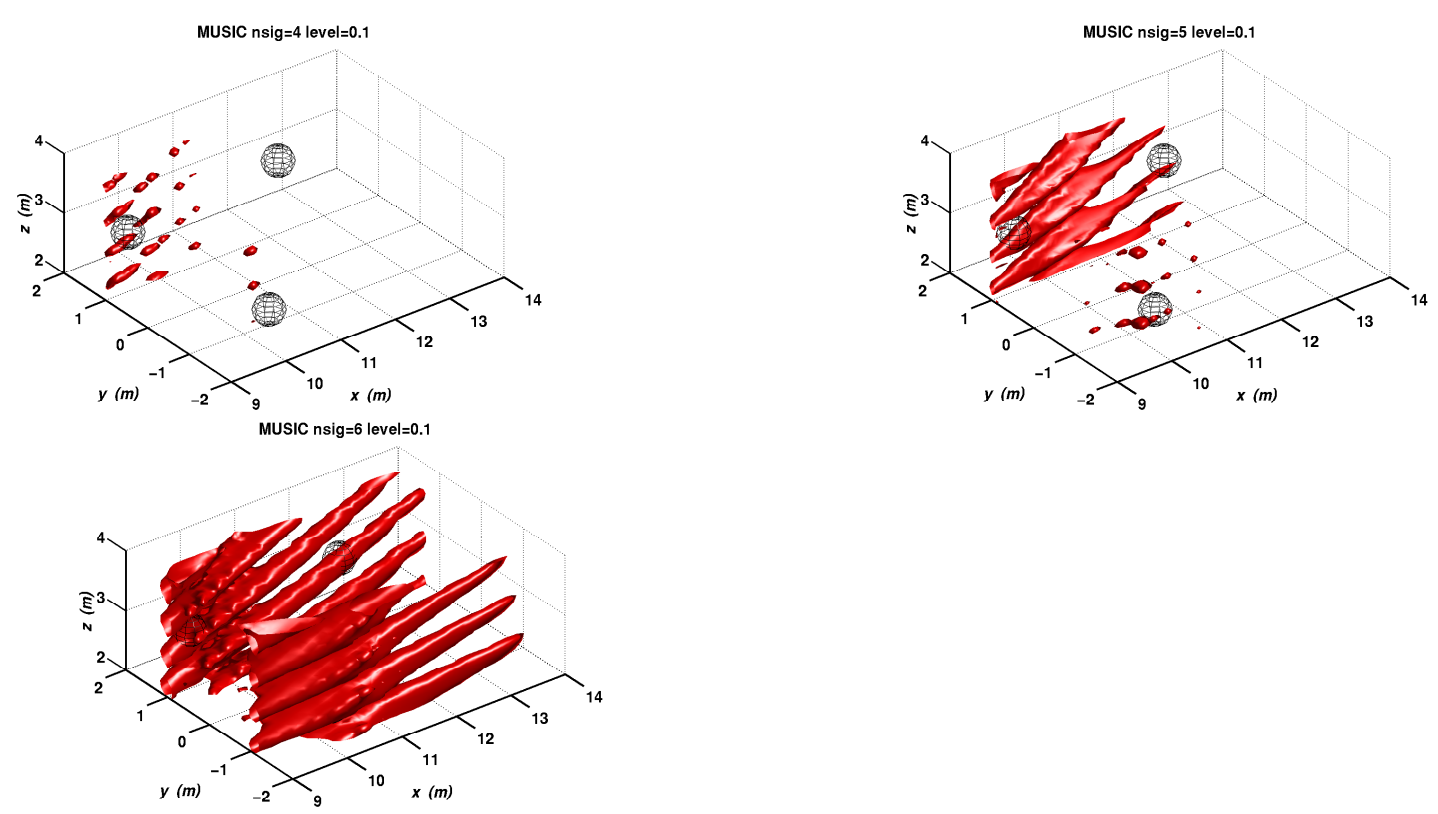

FIG. 20. Isosurfaces of the normalized MUSIC functional (10\% of maximum) using the ground plane Greens function for signal subspace dimensions of 4 (a), 5 (b), and 6(c) (case 1). Spheres are shown as an open meshes.

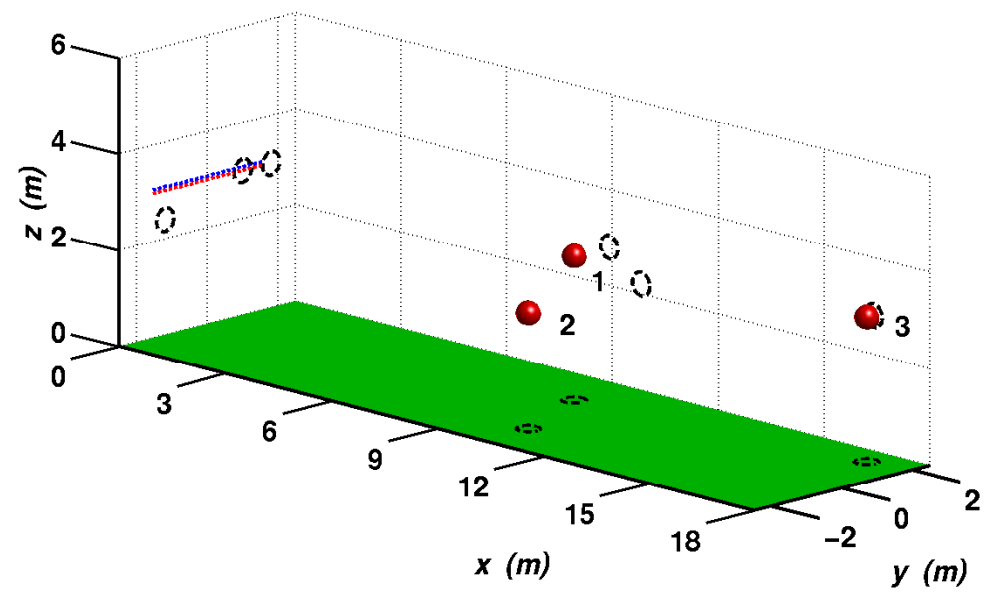

FIG. 21. Geometry of three spheres simulation - case 2. The linear array in the yz plane is composed of 21 transmit elements (red) and 21 receiver elements (blue). The $60 \mathrm{~cm}$ diameter spheres are centered at the points $(10.3-1.22 .4),(9.4,1.0,3.0)$, and $(16.9,1.8,3.0)$ meters. 


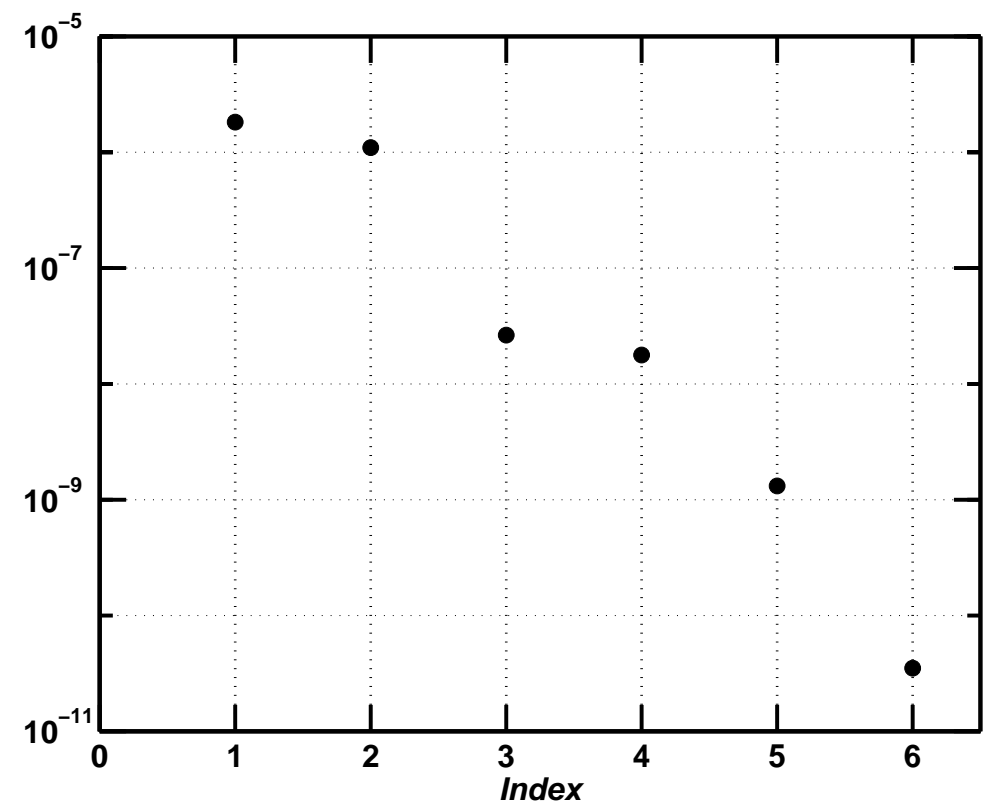

FIG. 22. First six singular values in the spectrum for three spheres - case 2. 

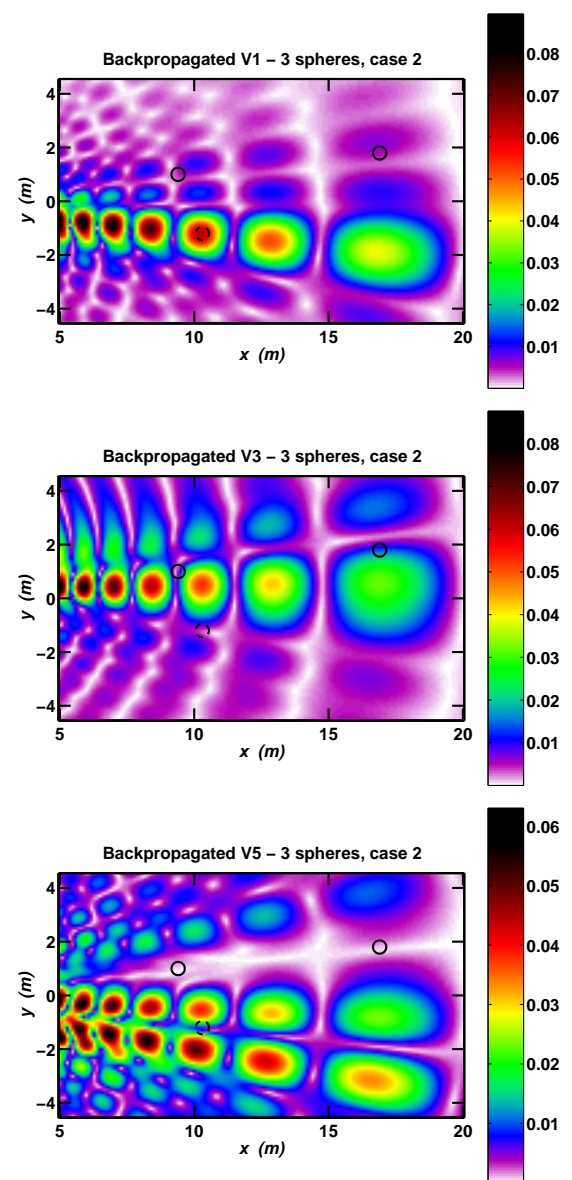
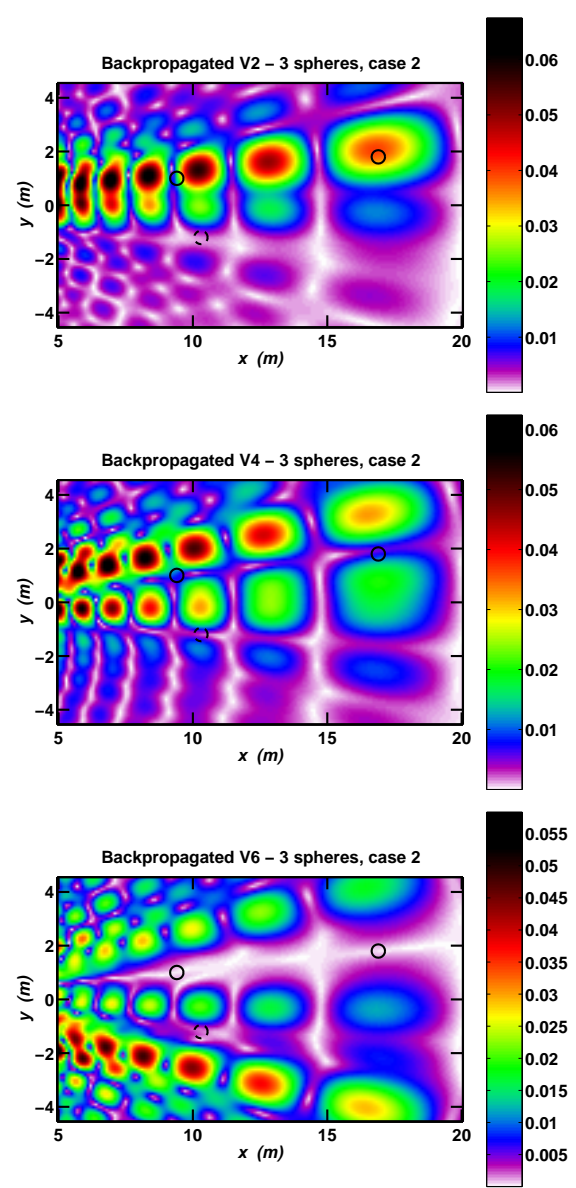

FIG. 23. Magnitude of the fields obtained by backpropagating the first (a), second (b), third (c), fourth (d), fifth (e), and sixth (f) singular vectors using the ground plane Greens function (case 2). The field is evaluated in the plane 3 meters above the ground. The intersections of the two spheres in the image plane are shown as black circles. The third sphere is centered $60 \mathrm{~cm}$ below the dashed circle in the image plane. 

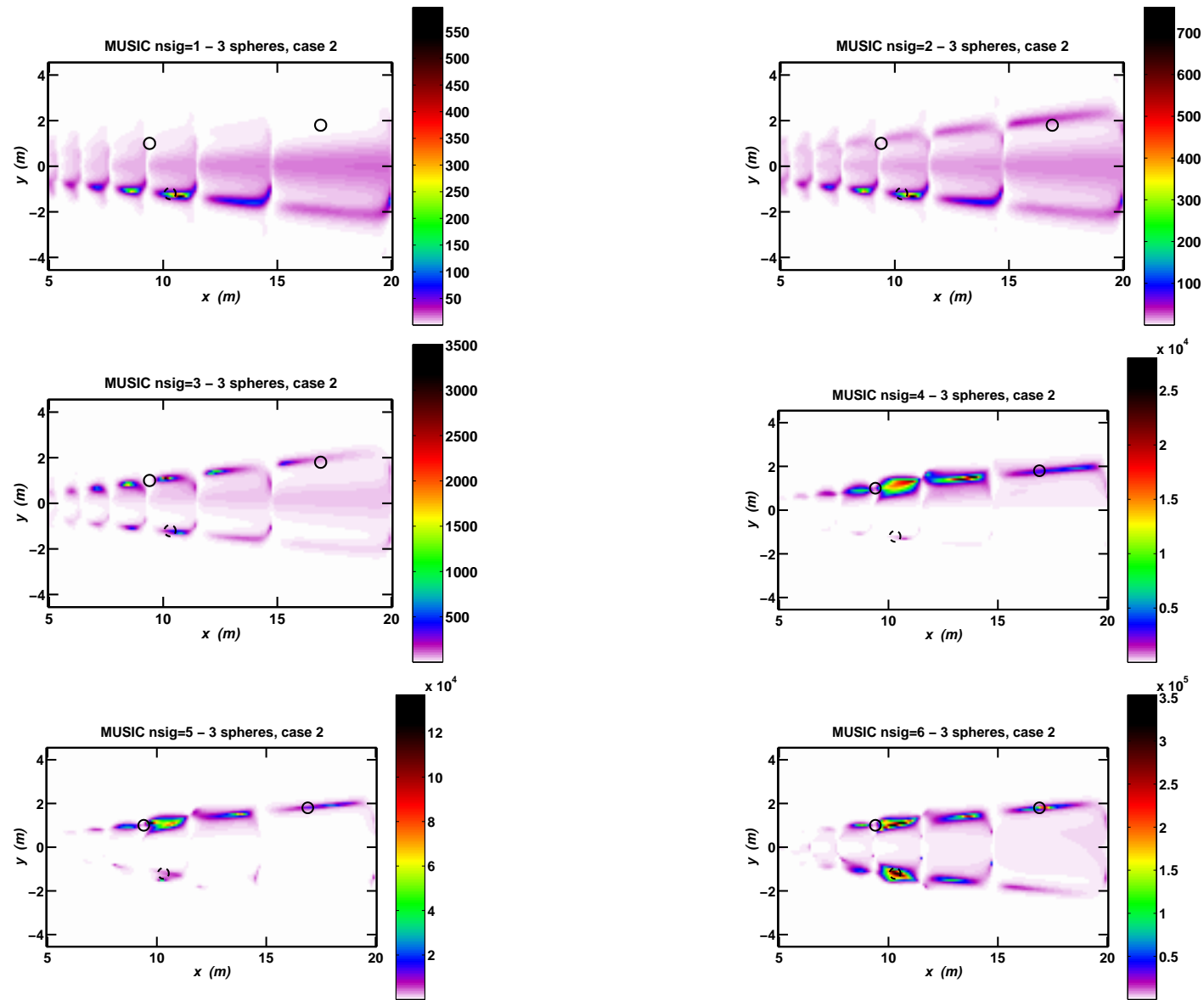

FIG. 24. Normalized MUSIC images using the ground plane Greens function for signal subspace dimensions of 1 (a), 2 (b), 3 (c), 4 (d), 5 (e), and 6 (f) evaluated in the plane 3 meters above the ground (case 2). The intersections of the two spheres in the image plane are shown as black circles. The third sphere is centered $60 \mathrm{~cm}$ below the dashed circle in the image plane. 

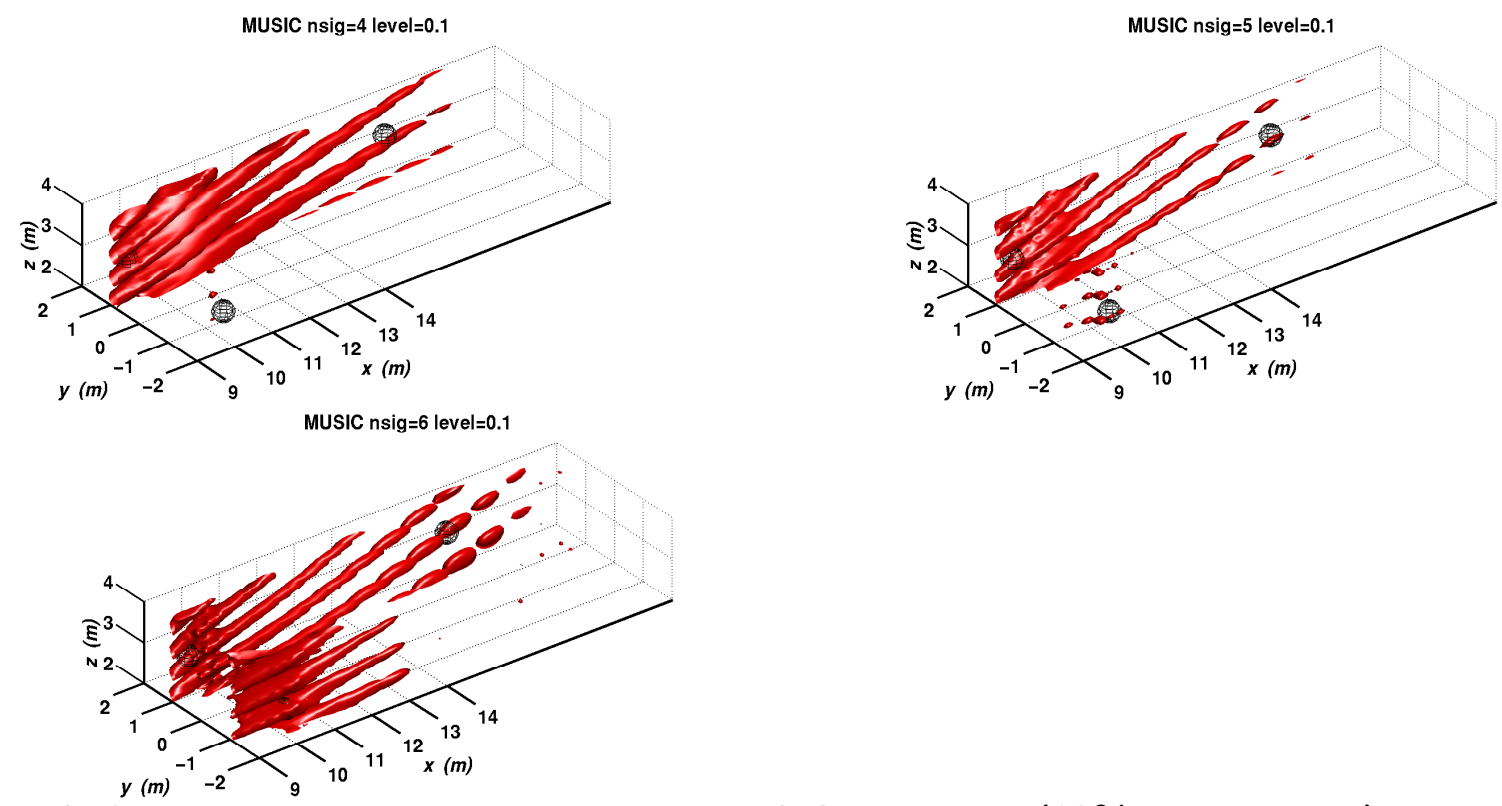

FIG. 25. Isosurfaces of the normalized MUSIC functional (10\% of maximum) using the ground plane Greens function for signal subspace dimensions of 4 (a), 5 (b), and 6(c) (case 2). Spheres are shown as an open meshes.

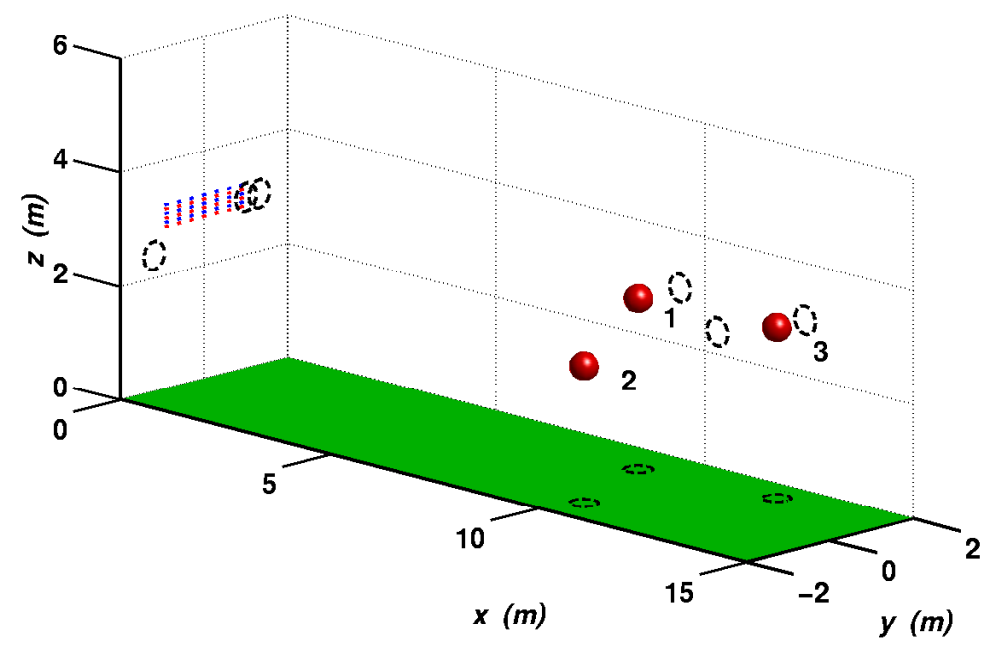

FIG. 26. Geometry of three spheres simulation - planar case. The planar array in the yz plane is composed of 21 transmit elements (red) and 21 receiver elements (blue). The $60 \mathrm{~cm}$ diameter spheres are centered at the points $(10.3-1.22 .4),(9.4,1.0,3.0)$, and $(12.4,1.32,3.0)$ meters (same as previous case 1 ). 


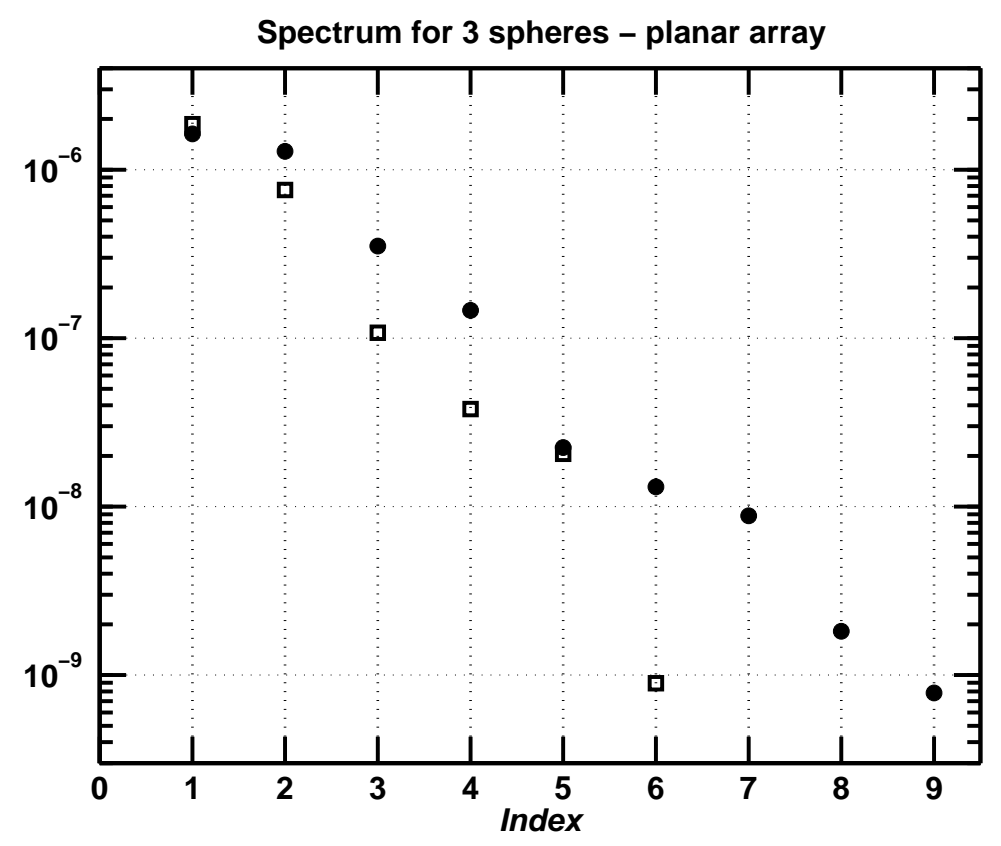

FIG. 27. First nine singular values in the spectra of three spheres for the linear array (squares) and the planar array (solid circles). 

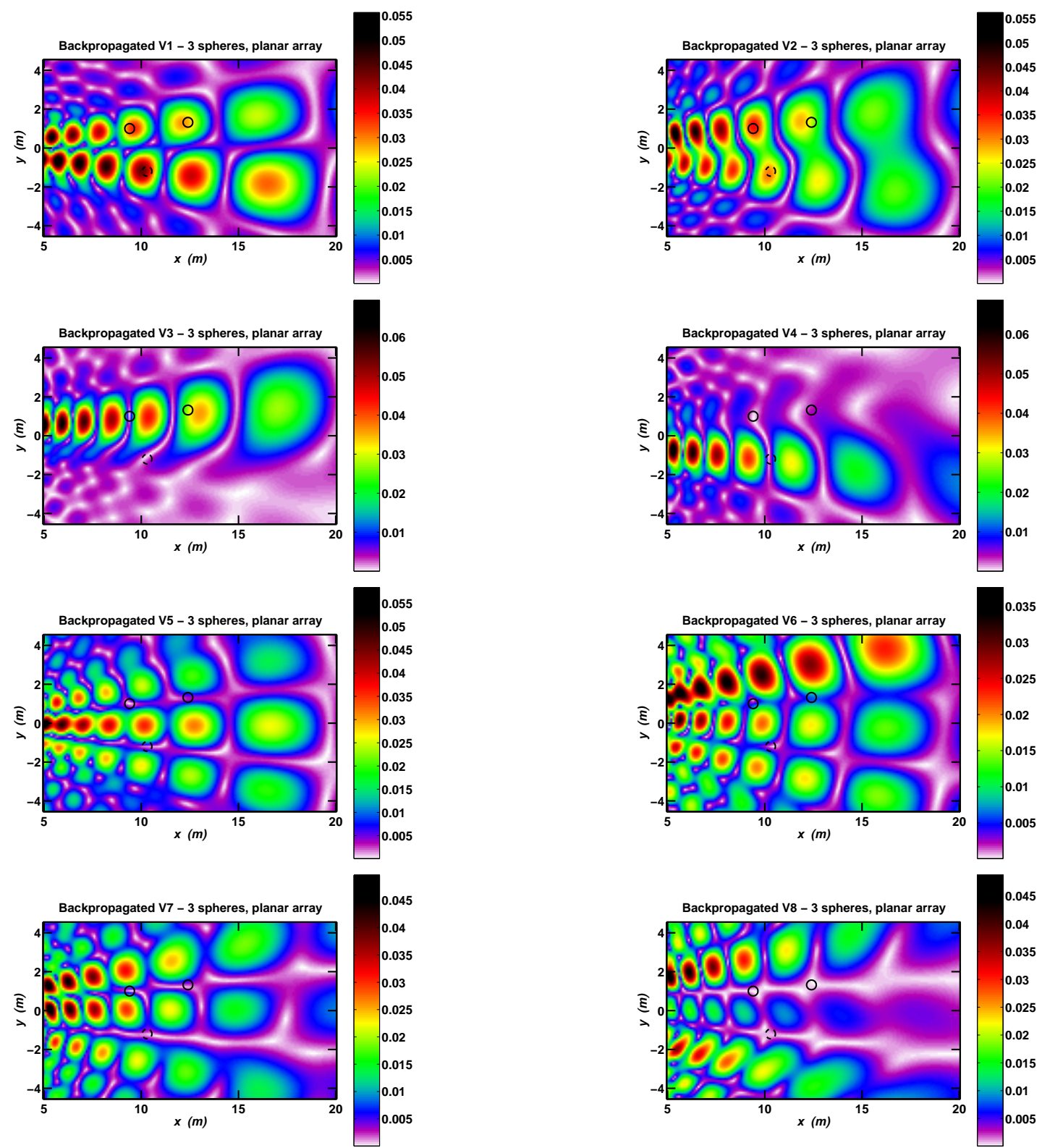

FIG. 28. Magnitude of the fields obtained by backpropagating the first (a), second (b), third (c), fourth (d), fifth (e), sixth (f), seventh (g), and eighth (h) singular vectors using the ground plane Greens function (planar array). The field is evaluated in the plane 3 meters above the ground. The intersections of the two spheres in the image plane are shown as black circles. The third sphere is centered $60 \mathrm{~cm}$ below the dashed circle in the image plane. 

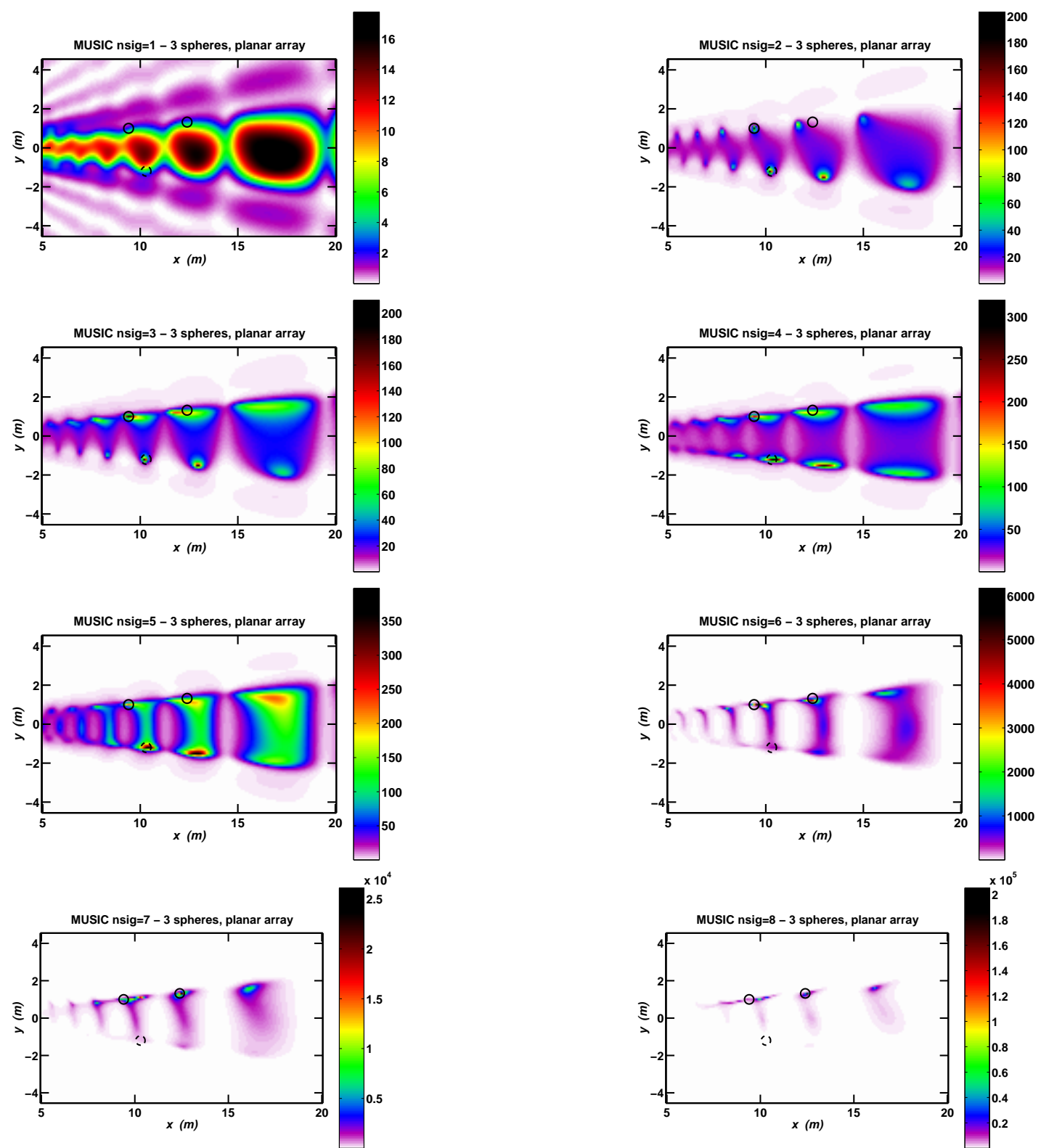

FIG. 29. Normalized MUSIC images using the ground plane Greens function for signal subspace dimensions of 1 (a), 2 (b), 3 (c), 4 (d), 5 (e), 6 (f), 7 (g), and 8 (h) evaluated in the plane 3 meters above the ground (planar array). The intersections of the two spheres in the image plane are shown as black circles. The third sphere is centered $60 \mathrm{~cm}$ below the dashed circle in the image plane. 

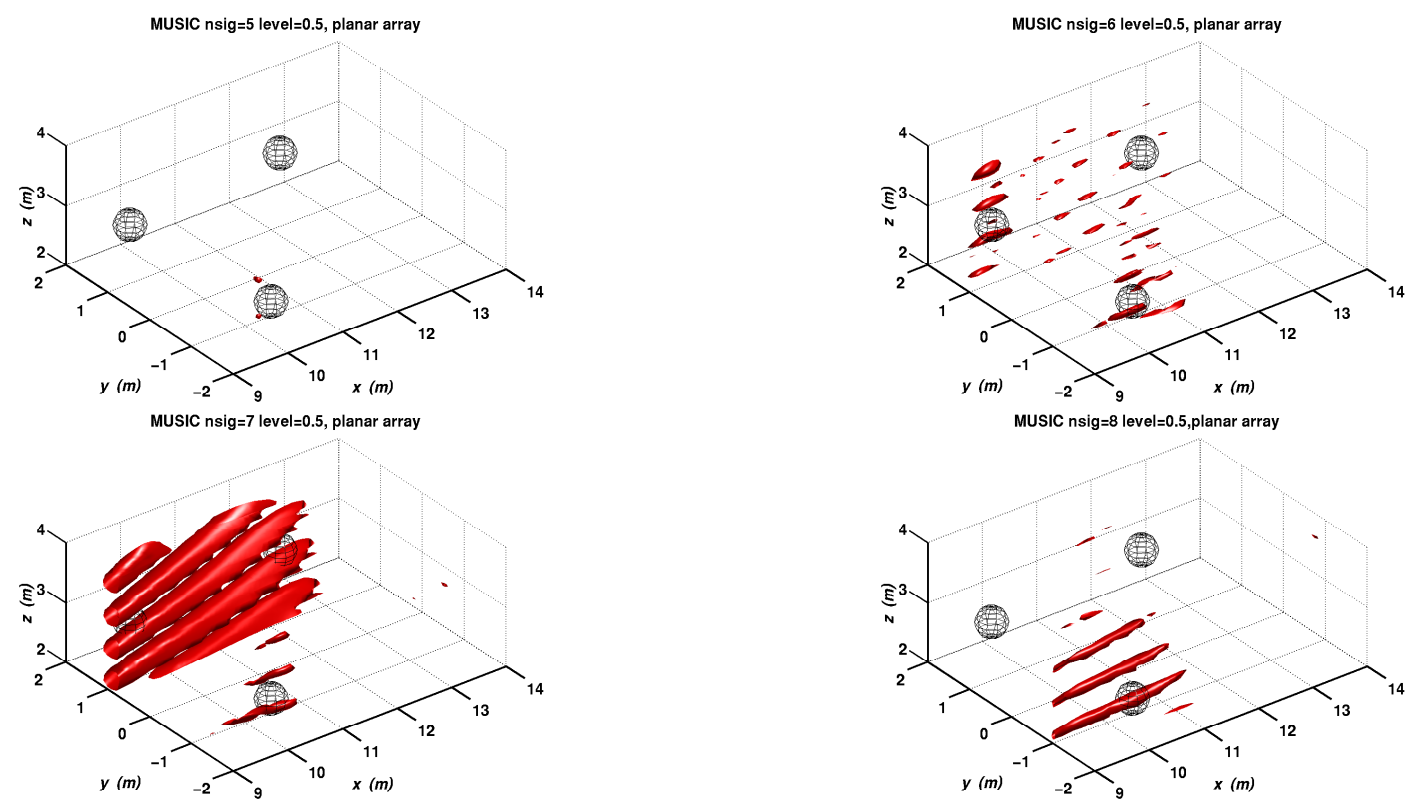

FIG. 30. Isosurfaces of the normalized MUSIC functional (50\% of maximum) using the ground plane Greens function for signal subspace dimensions of 5 (a), 6 (b), 7 (c), and 8 (d) (planar array). Spheres are shown as an open meshes. 